\title{
Theory Learning as Stochastic Search in a Language of Thought
}

\author{
Tomer D. Ullman ${ }^{\mathrm{a}, *}$, Noah D. Goodman ${ }^{\mathrm{b}}$, Joshua B. Tenenbaum ${ }^{\mathrm{a}}$ \\ ${ }^{a}$ Department of Brain and Cognitive Sciences, Massachusetts Institute of Technology, \\ Cambridge, USA \\ ${ }^{b}$ Department of Psychology, Stanford University, Stanford, USA
}

\begin{abstract}
We present an algorithmic model for the development of children's intuitive theories within a hierarchical Bayesian framework, where theories are described as sets of logical laws generated by a probabilistic context-free grammar. We contrast our approach with connectionist and other emergentist approaches to modeling cognitive development: while their subsymbolic representations provide a smooth error surface that supports efficient gradient-based learning, our symbolic representations are better suited to capturing children's intuitive theories but give rise to a harder learning problem, which can only be solved by exploratory search. Our algorithm attempts to discover the theory that best explains a set of observed data by performing stochastic search at two levels of abstraction: an outer loop in the space of theories, and an inner loop in the space of explanations or models generated by each theory given a particular dataset. We show that this stochastic search is capable of learning appropriate theories in several everyday domains, and discuss its dynamics in the context of empirical studies of children's learning.
\end{abstract}

Keywords: Bayesian models, MCMC, algorithms, language of thought, intuitive theory

\section{Introduction}

If a person should say to you "I have toiled and not found", don't believe. If they say "I have not toiled but found", don't believe. If they say "I have

\footnotetext{
*Corresponding author. Tel.: +1 6174523894

Email address: tomeru@mit.edu (Tomer D. Ullman)
} 
toiled and found", believe. - Rabbi Itz'hak, Talmud

For the Rabbis of old, learning was toil, exhausting work - a lesson which many scientists also appreciate. Over recent decades, scientists have toiled hard trying to understand learning itself: what children know when, and how they come to know it. How do children go from sparse fragments of observed data to rich knowledge of the world? From one instance of a rabbit to all rabbits, from occasional stories and explanations about a few animals to an understanding of basic biology, from shiny objects that stick together to a grasp of magnetism - children seem to go far beyond the specific facts of experience to structured interpretations of the world.

What some scientists found in their toil is themselves. It has been argued that children's learning is much like a kind of science, both in terms of the knowledge children create, its form, content, and function, and the means by which they create it. Children organize their knowledge into intuitive theories, abstract coherent frameworks that guide inference and learning within particular domains (Carey, 1985, 2009; Wellman \& Gelman, 1992; Gopnik \& Meltzoff, 1997; Murphy \& Medin, 1985). Such theories allow children to generalize from given evidence to new examples, make predictions and plan effective interventions on the world. Children even construct and revise these intuitive theories using many of the same practices that scientists do (Schulz, In press): searching for theories that best explain the data observed, trying to make sense of anomalies, exploring further and even designing new experiments that could produce informative data to resolve theoretical uncertainty, and then revising their hypotheses in light of the new data.

Consider the following concrete example of theory acquisition which we will return to frequently below. A child is given a bag of shiny, elongated, hard objects to play with, and finds that some pairs seem to exert mysterious forces on each other, pulling or pushing apart when they are brought near enough. These are magnets, but she doesn't know what that would mean. This is her first exposure to the domain. To make matters more interesting, and more like the situation of early scientists exploring the phenomena of magnetism in nature, suppose that all of the objects have an identical metallic appearance, but only some of them are magnetic, and only a subset of those are actually magnets (permanently magnetized). She may initially be confused trying to figure out what interacts with what, but like a scientist developing a first theory, after enough exploration and experimentation, she might start to sort the objects into groups based on similar behaviors or 
similar functional properties. She might initially distinguish two groups, the magnetic objects (which can interact with each other) and the nonmagnetic ones (which do not interact). Perhaps then she will move on to subtler distinctions, noticing that this very simple theory doesn't predict everything she observes. She could distinguish three groups, separating the permanent magnets from the rest of the magnetic objects as well as from the nonmagnetic objects, and recognizing that there will only be an interaction if at least one of the two magnetic objects brought together is a permanent magnet. With more time to think and more careful observation, she might even come to discover the existence of magnetic poles and the laws by which they attract or repel when two magnets are brought into contact. These are but three of a large number of potential theories, varying in complexity and power, that a child could entertain to explain her observations and make predictions about unseen interactions in this domain.

Our goal here is to explore computational models for how children might acquire and revise an intuitive theory such as this, on the basis of domain experience. Any model of learning must address two kinds of questions: what, and how? Which representations can capture the form and content of what the learner comes to know, and which principles or mechanisms can explain how the learner comes to know it, moving from one state of knowledge to another in response to observed data? The main new contribution of this paper addresses the 'how' question. We build on much recent work addressing the 'what' question, which proposes to represent the content of children's intuitive theories as probabilistic generative models defined over hierarchies of structured symbolic representations (Tenenbaum et al., 2006, 2011; Kemp et al., 2008b). Previously the 'how' question has been addressed only at a very high level of abstraction, if at all: the principles of Bayesian inference explain how an ideal learner can successfully identify an appropriate theory, based on maximizing the posterior probability of a theory given data (as given by Bayes' rule). But Bayes' rule says nothing about the processes by which a learner could construct such a theory, or revise it in light of evidence. Here our goal is to address the 'how' of theory construction and revision at a more mechanistic, process level, exploring cognitively realistic learning algorithms. Put in terms of Marr's three levels of analysis (Marr, 1982), previous Bayesian accounts of theory acquisition have concentrated on the level of computational theory, while here we move to the algorithmic level of analysis, with the aim of giving a more plausible, practical and experimentally fertile view of children's developmental processes within the 
Bayesian paradigm.

Our work here aims to explain two challenges of theory acquisition in algorithmic terms. First is the problem of making learning work: getting the world right, as reliably as children do. As any scientist can tell you, reflecting on their own experiences of toil, the 'how' of theory construction and revision is nontrivial. The process is often slow, painful, a matter of starts and stops, random fits and bursts, missteps and retreats, punctuated by occasional moments of great insight, progress and satisfaction - the flashes of 'Aha!' and 'Eureka!'. And as any parent will tell you, children's cognitive development often seems to have much the same character. Different children make their way to adult-like intuitive theories at very different paces. Transitions between candidate theories often appear somewhat random and unpredictable at a local level, prone to backtracking or "two steps forward, one step back" behavior (Siegler \& Chen, 1998). Yet in core domains of knowledge, and over long time scales, theory acquisition is remarkably successful and consistent: different children (at least within a common cultural context of shared experience) tend to converge on the same knowledge structures, knowledge that is much closer to a veridical account of the world's causal structure than the infant's starting point, and they follow predictable trajectories along the way (Carey, 2009; Gopnik \& Meltzoff, 1997; Wellman et al., 2011).

Our first contribution is an existence proof to show how this kind of learning could work - a model of how a search process with slow, fitful and often frustrating stochastic dynamics can still reliably get the world right, in part because of these dynamics, not simply in spite of them. The process may not look very algorithmic, in the sense of familiar deterministic algorithms such as those for long division, finding square roots, or sorting a list, or what cognitive scientists typically think of as a "learning algorithm", such as the backpropagation algorithm for training neural networks. Our model is based on a Monte Carlo algorithm, which makes a series of randomized (but not entirely random) choices as part of its execution. These choices guide how the learner explores the space of theories to find those that best explain the observed data - influenced by, but not determined by, the data and the learner's current knowledge state. We show that such a Monte Carlo exploratory search yields learning results and dynamics qualitatively similar to what we see in children's theory construction, for several illustrative cases.

Our second challenge is to address what could be called the "hard problem" of theory learning: learning a system of concepts that cannot be simply expressed as functions of observable sense data or previously available con- 
cepts - knowledge that is not simply an extension or addition to what was known before, but that represents a fundamentally new way to think. Developmental psychologists, most notably Carey (2009), have long viewed this problem of conceptual change or theory change as one of the central explanatory challenges in cognitive development. To illustrate, consider the concepts of "magnet" or "magnetic object" or "magnetic pole" in our scenario above, for a child first learning about them. There is no way to observe an object on its own and decide if it falls under any of these concepts. There is no way to define or describe either "magnet" or "magnetic object" in purely sensory terms (terms that do not themselves refer to the laws and concepts of magnetism), nor to tell the difference between a "north" and a "south" magnetic pole from perception alone. How then could these notions arise? They could be introduced in the context of explanatory laws in a theory of magnetism, such as "Two objects will interact if both are magnetic and at least one is a magnet", or "Magnets have two poles, one of each type, and opposite types attract while like types repel." If we could independently identify the magnets and the magnetic objects, or the two poles of each magnetic object and their types, then these laws would generate predictions that could be tested on observable data. But only in virtue of these laws' predictions can magnets, magnetic objects, or magnetic poles even be identified or made meaningful. And how could one even formulate or understand one of these laws without already having the relevant concepts?

Theory learning thus presents children with a difficult joint inference task - a "chicken-and-egg" problem - of discovering two kinds of new knowledge, new concepts and new laws, which can only be made sense of in terms of each other: the laws are defined over the concepts, but the concepts only get their meaning from the roles they play in the laws. If learners do not begin with either the appropriate concepts or the appropriate laws, how can they end up acquiring both successfully? This is also essentially the challenge that philosophers have long studied of grounding meaning in conceptual role or inferential role semantics (Block, 1986; Harman, 1975, 1982; Field, 1977; Fodor \& Lepore, 1991). Traditional approaches to concept learning in psychology do not address this problem, nor do they even attempt to (Bruner et al., 1956; Smith \& Medin, 1981; Rogers \& McClelland, 2004). The elusiveness of a satisfying solution has led some scholars, most famously Jerry Fodor, to a radical skepticism on the prospects for learning genuinely new concepts, and a view that most concepts must be innate in some nontrivial way (Fodor, 1975, 1980). Carey (2009) has proposed a set of informal "bootstrapping" 
mechanisms for how human learners could solve this problem, but no formal model of bootstrapping exists for theory learning, or concept learning in the context of acquiring novel theories.

We will argue that the chicken-and-egg problem can be solved by a rational learner but must be addressed in algorithmic terms to be truly satisfying: a purely computational-level analysis will always fail for the Fodorian skeptic, and will fail to make contact with the crux of the bootstrapping problem as Carey (2009) frames it, since for the ideal learner the entire space of possible theories, laws and concepts, is in a sense already available from the start. An algorithmic implementation of that same ideal learning process can, however, introduce genuinely new concepts and laws in response to observed data. It can provide a concrete solution to the problem of how new concepts can be learned and can acquire meaning in a theory of inferential role semantics. Specifically, we show how a Monte Carlo search process defined over a hierarchically structured Bayesian model can effectively introduce new concepts as blank placeholders in the context of positing a new candidate explanatory law or extending an existing law. The new concept is not expressed in terms of pre-existing concepts or observable data; rather it is posited as part of a candidate explanation, together with pre-existing concepts, for observed data. In testing the candidate law's explanatory power, the new concepts are given a concrete interpretation specifying which entities they are most likely to apply to, assuming the law holds. If the new or modified law turns out to be useful - that is, if it leads to an improved account of the learner's observations, relative to their current theory - the law will tend to be retained, and with it, the new concept and its most likely concrete grounding.

The rest of the paper is organized as follows. We first present a nontechnical overview of the "what" and "how" of our approach to theory learning, and contrast it with the most well-known alternatives for modeling cognitive development based on connectionism and other emergentist paradigms. We then describe our approach more technically, culminating in a Markov Chain Monte Carlo (MCMC) search algorithm for exploring the space of candidate theories based on proposing random changes to a theory and accepting probabilistically those changes that tend to improve the theory. We highlight two features that make the dynamics of learning more efficient and reliable, as well as more cognitively plausible: a prior that proposes new theoretical laws drawn from law templates, biasing the search towards laws that express canonical patterns of explanation useful across many domains, and a process of annealing the search that reduces the amount of random exploration 
over time. We study the algorithm's behavior on two case studies of theory learning inspired by everyday cognitive domains: the taxonomic organization of object categories and properties, and a simplified version of magnetism. Finally, we explore the dynamics of learning that arise from the interaction between computational-level and algorithmic-level considerations: how theories change both as a function of the quantity and quality of the learner's observations, and as a function of the time course of the annealing-guided search process, which suggests promising directions for future experimental research on children's learning.

\section{A nontechnical overview}

A proposal for what children learn and a proposal for how they learn it may be logically independent in some sense, but the two are mutually constraining. Richer, more structured accounts of the form and content of children's knowledge tend to pose harder learning challenges, requiring learning algorithms that are more sophisticated and more costly to execute. As we explain below, our focus on explaining the origins of children's intuitive theories leads us to adopt relatively rich abstract forms of knowledge representations, compared to alternative approaches to modeling cognitive development, such as connectionism. This leaves us with relatively harder learning challenges connectionists might argue, prohibitively large. But we see these challenges as inevitable: Sooner or later, computational models of development must face them. Perhaps for the first time, we can now begin to see what their solution might look like, by bringing together recent ideas for modeling the form and content of theories as probabilistic generative models over hierarchies of symbolic representations (Katz et al., 2008; Kemp et al., 2008a; Goodman et al., 2011) with tools for modeling the dynamics of learning as exploratory search based on stochastic Monte Carlo algorithms.

\subsection{The 'What': Modeling the form and content of children's theories as hierarchical probabilistic models over structured representations}

As a form of abstract knowledge, an intuitive theory is similar to the grammar of a language (Tenenbaum et al., 2007): The concepts and laws of the theory can be used to generate explanations and predictions for an infinite (though constrained) set of phenomena in the theory's domain. We follow a long tradition in cognitive science and artificial intelligence of representing such knowledge in terms of compositional symbol systems, specifically 
predicate logic that can express a wide range of possible laws and concepts (Fodor, 1975; Fodor \& Pylyshyn, 1988; Russell \& Norvig, 2009). Embedding this symbolic description language in a hierarchical probabilistic generative model lets us bring to bear the powerful inductive learning machinery of Bayesian inference, at multiple levels of abstraction (Griffiths et al., 2010; Tenenbaum et al., 2011).

Figure 1 illustrates this framework. We assume a domain of cognition is given, comprised of one or more systems of entities and their relations, each of which gives rise to some observed data. The learner's task is to build a theory of the domain: a set of abstract concepts and explanatory laws that explain the observed data for each system in that domain. The learner is assumed to have a hypothesis space of possible theories generated by (and constrained by) some "Universal Theory". We formalize this Universal Theory as a probabilistic generative grammar, essentially a probabilistic version of a language of thought (Fodor, 1975). Within this universal language, the learner constructs a specific theory that can be thought of as a more specific language for explaining the phenomena of the given domain.

In principle, an ideal learner should consider all possible theories expressible in the language of thought and weigh them against each other in light of observed evidence. In practice, there are infinitely many candidate theories and it will be impossible to explicitly consider even a small fraction of them. Explaining how a learner proposes specific candidate theories for evaluation is a task for our algorithmic-level account (see below under 'How').

Candidate theories are evaluated using Bayes' rule to assess how likely they are to have generated the observed data. Bayes' rule scores theories based on the product of their prior probabilities and their likelihoods. The prior reflects the probability of generating the laws and concepts of a theory a priori from the generative grammar, independent of any data to be explained. The likelihood measures the probability of generating the observed data given the theory, independent of the theory's plausibility. Occam's razor-like considerations emerge naturally from a Bayesian analysis: the prior will be highest for the simplest theories, whose laws can be generated with the fewest number of a priori stipulations, while the likelihood will be highest for theories whose laws allow a domain to be described accurately and compactly, generating the observed data with a spare set of minimal facts.

The fit of a theory to data cannot be evaluated directly; its laws express the abstract principles underlying a domain but no specific expectations 
about what is true or false. One level below the theory in the hierarchical framework, the learner posits a logical model of each observed system in the domain. The logical model, or "model" for short, specifies what is true of the entities in a particular system in ways consistent with and constrained by the theory's abstract laws. Each model can be thought of as one particular concrete instantiation of the abstract theory. It generates a probability distribution over possible observations for the corresponding system, and it can be scored directly in terms of how well those predictions fit the actual data observed.

As a concrete example of this framework, consider again the child learning about the domain of magnetism. She might begin by playing with a few pieces of metal and notice that some of the objects interact, exerting strange pulling or pushing forces on each other. She could describe the data directly, as "Object $a$ interacts with object $j$ ", "Object $i$ interacts with object $\jmath$ ", and so on. Or she could form a simple theory, in terms of abstract concepts such as magnet, magnetic object and non-magnetic object, and laws such as "Magnets interact with other magnets", "Magnets interact with magnetic objects", and "Interactions are symmetric". It is important to note that terms like magnet convey no actual information about the object, and they are simply labels. Systems in this domain correspond to specific subsets of objects, such as the set of objects $a, \ldots, i$ in Figure 1. A model of a system specifies the minimal facts needed to apply the abstract theory to the system, in this case which objects are magnetic, which are magnets, and which are non-magnetic. From these core facts the laws of the theory determine all other true facts - in our example, this means all the pairwise interactions between the objects: e.g., objects $i$ and $j$, being magnets, should interact, but $i$ and $e$ should not, because the latter is non-magnetic. Finally, the true facts generate the actual data observed by the learner via a noisy sampling process, e.g. observing a random subset of the object pairs that interact, and occasionally misperceiving an object's identity or the nature of an interaction.

While the abstract concepts in this simplified magnetism theory are attributes of objects, more complex relations are possible. Consider for example a theory of taxonomy, as in Collins and Quillian's classic model of semantic memory as an inheritance hierarchy (Collins \& Quillian, 1969). Here the abstract concepts are $i s_{-} a$ relations between categories and has_a relations between categories and properties. The theory underlying taxonomy has two basic laws: "The $i s_{-} a$ relation is transitive" and "The has_a relation inherits down $i s \_a$ relations" (laws 3 and 4 on the "Taxonomy" column of Figure 
1). A system consists of a specific set of categories and properties, such as salmon, eagle, breathes, can fly, and so on. A model specifies the minimal $i s_{-} a$ and $h a s_{-} a$ relations, typically corresponding to a tree of $i s_{-} a$ relations between categories with properties attached by has_a relations at the broadest category they hold for: e.g., "A canary is a bird", "A bird is an animal", "An animal can breathe", and so on. The laws then determine that properties inherit down chains of $i s_{-} a$ relations to generate many other true facts that can potentially be observed, e.g., "A canary can breathe".

The analogy between learning a theory for a domain and learning a grammar for a natural language thus extends down through all levels of the hierarchy of Figure 1. A logical model for a system of observed entities and relations can be thought of as a parse of that system under the grammar of the theory, just as the theory itself can be thought of as a parse of a whole domain under the grammar of the universal theory. In our hierarchical Bayesian framework, theory learning is the problem of searching jointly for the theory of a domain and models of each observed system in that domain that together best parse all the observed data. ${ }^{1}$

Previous applications of grammar-based hierarchical Bayesian models have shown how, given sufficient evidence and a suitable theory grammar, an ideal Bayesian learner can identify appropriate theories in domains such as causality (Griffiths et al., 2010; Goodman et al., 2011), kinship and other social structures (Kemp et al., 2008a), and intuitive biology (Tenenbaum et al., 2007). While our focus in this paper is the algorithmic level - the dynamics of how learners can search through a space of theories - we have found that endowing our theory grammars with one innovation greatly improves their algorithmic tractability. We make the grammar more likely to generate theories with useful laws by equipping it with law templates, or forms of laws that capture canonical patterns of coherent explanation arising in many domains. For example, law templates might suggest explanations for when an observed relation $r(X, Y)$ holds between entities $X$ and $Y$ (e.g., $X$ attracts $Y, X$ activates $Y, X$ has $Y$ ) in terms of latent attributes of the objects, $f(X)$ and $g(Y)$, or in terms of some other relation $s(X, Y)$ that holds between them, or some combination thereof: perhaps $r(X, Y)$ holds if $f(X)$ and $s(X, Y)$ are

\footnotetext{
${ }^{1}$ The idea of hierarchical Bayesian grammar induction, where the prior on grammars is itself generated by a grammar (or "grammar grammar"), dates back at least to the seminal work of Feldman and colleagues (Feldman et al., 1969).
} 
both true. Explanatory chains introducing novel objects are also included among the templates: perhaps $r(X, Y)$ holds if there exists a $Z$ such that $s(X, Z)$ and $s(Z, Y)$ hold. As we explain below, making these templates explicit in the grammar makes learning both more cognitively plausible and much faster.

The most familiar computational alternative to structured Bayesian accounts of cognitive development are connectionist models, and other emergentist approaches (McClelland et al., 2010). Instead of representing children's abstract knowledge in terms of explicit symbol systems, these approaches attribute abstract knowledge to children only implicitly as an 'emergent' phenomenon that arises in a graded fashion from interactions among more concrete, lower-level non-symbolic elements - often inspired loosely by neuroscience. Dynamical systems models view the nervous system as a complex adaptive system evolving on multiple timescales, with emergent behavior in its dynamics. Connectionist models view children's knowledge as embedded in the strengths of connections between many neuron-like processing units, and treat development as the tuning of these strengths via some experience-dependent adjustment rule. Connectionists typically deny that the basic units of traditional knowledge representation - objects, concepts, predicates, relations, propositions, rules and other symbolic abstractions are appropriate for characterizing children's understanding of the world, except insofar as they emerge as approximate higher-level descriptions for the behavior dictated by a network's weights.

While emergentist models have been well-received in some areas of development, such as the study of motor and action systems (McClelland et al., 2010), emergentist models of the structure and origins of abstract knowledge (Rogers \& McClelland, 2004) have not been widely embraced by developmentalists studying children's theories (Gopnik \& Meltzoff, 1997; Carey, 2009). There is every reason to believe that explicit symbolic structure is just as important for children's intuitive theories as for scientists' more formal theories - that children, like scientists, cannot adequately represent the underlying structure of a domain such as physics, psychology or biology simply with a matrix of weights in a network that maps a given set of inputs to a given set of outputs. Children require explicit representations of abstract concepts and laws in order to talk about their knowledge in natural language, and to change and grow their knowledge through talking with others; to reason causally in order to plan for the future, explain the past, or imagine hypothetical situations; to apply their knowledge in novel settings to solve problems 


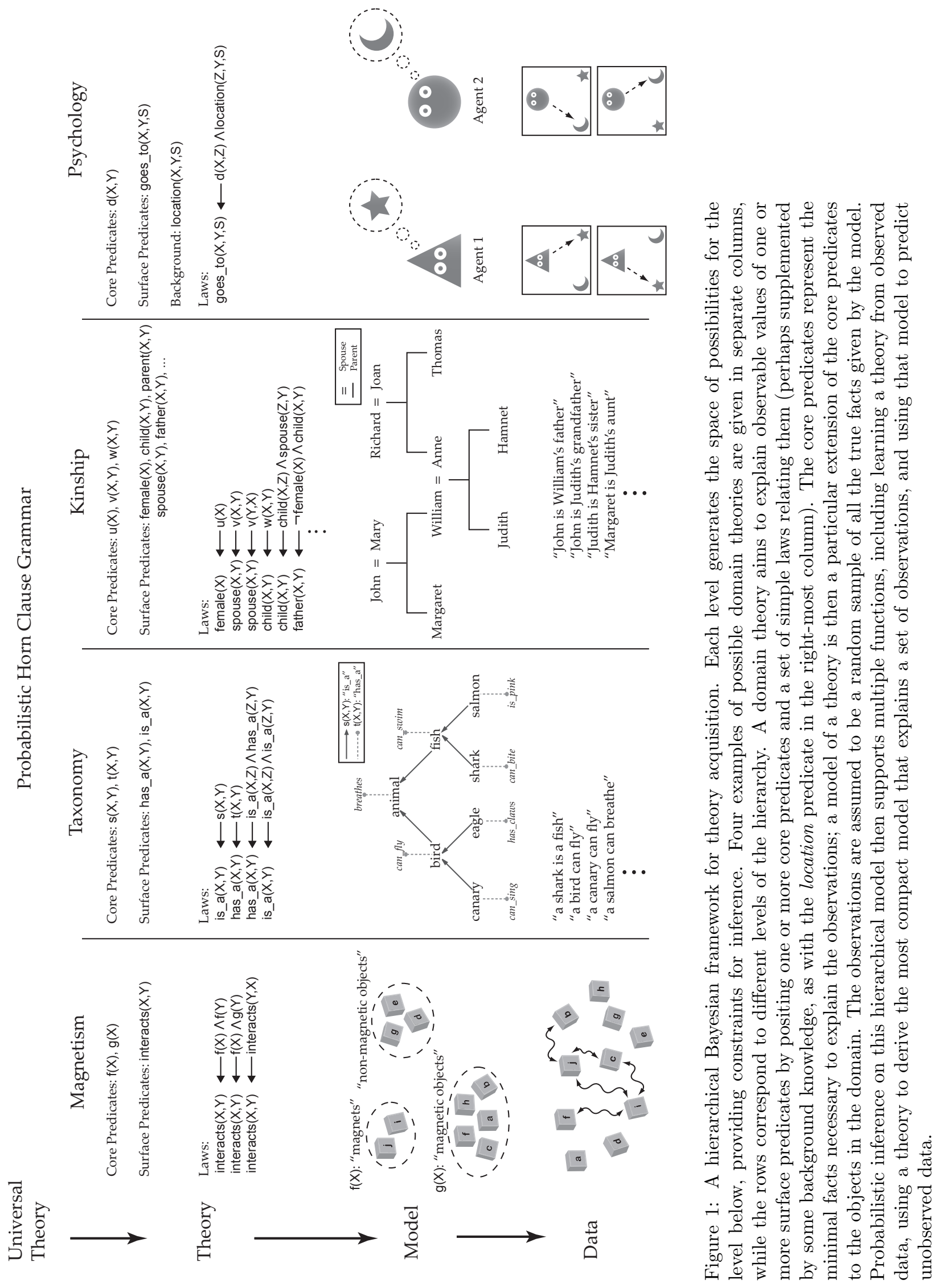


that they have never before encountered; and to compose abstractions recursively, as in forming beliefs about others' beliefs about the physical world and how those beliefs might be different than one's own.

Despite these limitations, connectionist models have been appealing to developmentalists who emphasize the processes and dynamics of learning more than the nature of children's knowledge representations (Shultz, 2003; McClelland et al., 2010). This appeal may come from the fact that when we turn from the 'what' to the 'how' of children's learning, connectionist models have a decided advantage: learning in connectionist systems appears much better suited to practical algorithmic formulation, and much more tractable, relative to structured probabilistic models or any explicitly symbolic approach. As we explain below, making the 'how' of learning plausible and tractable may be the biggest challenge facing the structured probabilistic approach.

\subsection{The 'How': Modeling the dynamics of children's theory learning as stochas- tic (Monte Carlo) exploratory search}

It is helpful to imagine the problem children face in learning as that of moving over a "knowledge landscape", where each point represents a possible state of knowledge and the height of that point reflects the value of that knowledge-state - how well it allows the child to explain, predict, and act on their world. Such a picture is useful in showing some of the differences between our approach to cognitive development and the connectionist and emergentist alternatives, and it highlights the much more serious 'how' challenge that confronts structured probabilistic models.

Viewed in landscape terms (Figure 2), connectionist models typically posit that children's knowledge landscape is continuous and smooth, and this matters greatly for the mechanisms and dynamics of learning. Learning consists of traversing a high-dimensional real-valued "weight space", where each dimension corresponds to the strength of one connection in a neural network. Figure 2 depicts only a two-dimensional slice of the much higher dimensional landscape corresponding to the three-layer network shown. The height of the landscape assigned to each point in weight space - each joint setting of all the network's weights - measures how well the network explains observed data in terms of an error or energy function, such as a sum-of-squared-error expression. The topology of these landscapes is simple and uniform: at any point of the space, one can always move along any dimension independently of every other, and changing one parameter has no effect on any other. The geometry is also straightforward: neighboring states, separated by small changes in 
the weights or parameters, typically yield networks with very similar inputoutput functionality. Thus a small move in any direction typically leads to only a small rise or fall in the error or energy function.

This geometry directly translates into the dynamics of learning: the Hebb rule, the Delta Rule, Backpropagation and other standard weight-adjustment rules (Mcclelland \& Rumelhart, 1986) can be seen as implementing gradient descent - descending the error or energy landscape by taking small steps along the steepest direction - and it can be proven that this dynamic reliably takes the network to a local minimum of error, or a locally best fitting state of knowledge. In certain cases, particularly of interest in contemporary machine learning systems (Bishop, 2006), the error landscape can be designed to have a geometric property known as convexity, ensuring that any local minimum is also a global minimum and thus that the best possible learning end-state can be achieved using only local weight-adjustment rules based on gradient descent. Thus learning becomes essentially a matter of "rolling downhill", and is just as simple. Even in cases where there are multiple distinct local minima, connectionist learning can still draw on a powerful toolkit of optimization methods that exploit the fact that the landscape is continuous and smooth to make learning relatively fast, reliable and automatic.

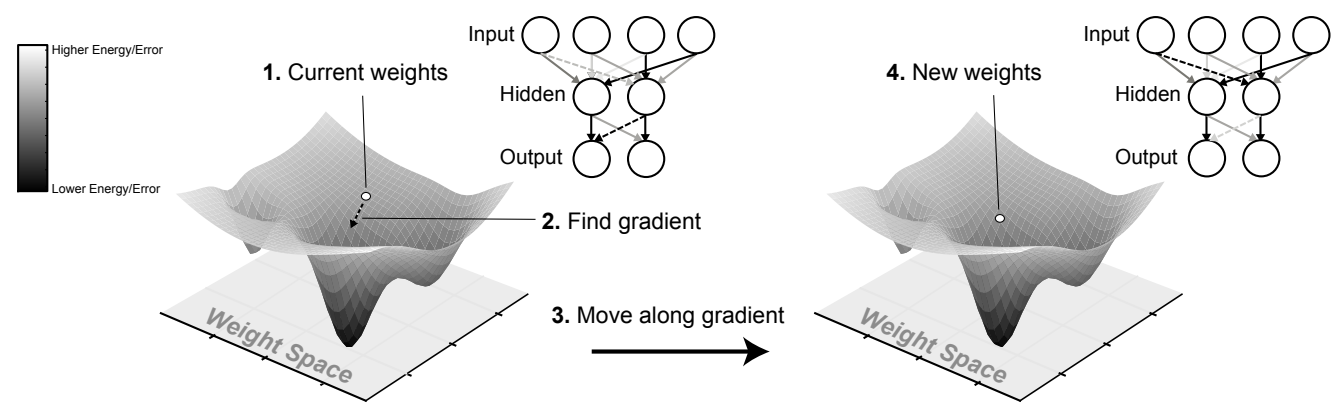

Figure 2: A hypothetical neural network and a weight space spanning the possible values of two particular connections. Steps 1-4 show the sequence of a learning algorithm in such a space: the calculation of a gradient and the move to a lower point. This corresponds to a shift in the network's connection weights and a smaller error on the output.

Now consider the landscape of theory learning from the perspective of our structured Bayesian approach, and it should become clear how much more difficult the problem is (Figure 3). Each point on the landscape now represents a candidate domain theory expressed in terms of one or more 
laws in first-order logic and one or more abstract concepts indicated by a blank predicate (e.g., $f(X), g(X)$ ). Two possibilities for a simple theory of magnetism are shown, labeled Theory B and Theory $\mathrm{C}$ (these will be explained in much greater detail below). The height of the surface at a given point represents how well the corresponding theory is supported by the observed data, which we measure as the Bayesian posterior probability. (Note that in contrast to Figure 2, where "lower is better", here "higher is better", and the goal is to seek out maxima of the landscape, not minima.) Unlike the weight space shown in Figure 2, this portrait of a "theory space" as two-dimensional is only metaphorical: it is not simply a lower-dimensional slice of a higher-dimensional space. The space of theories in a language of thought is infinite and combinatorially structured with a neighborhood structure that is impossible to visualize faithfully on a page.
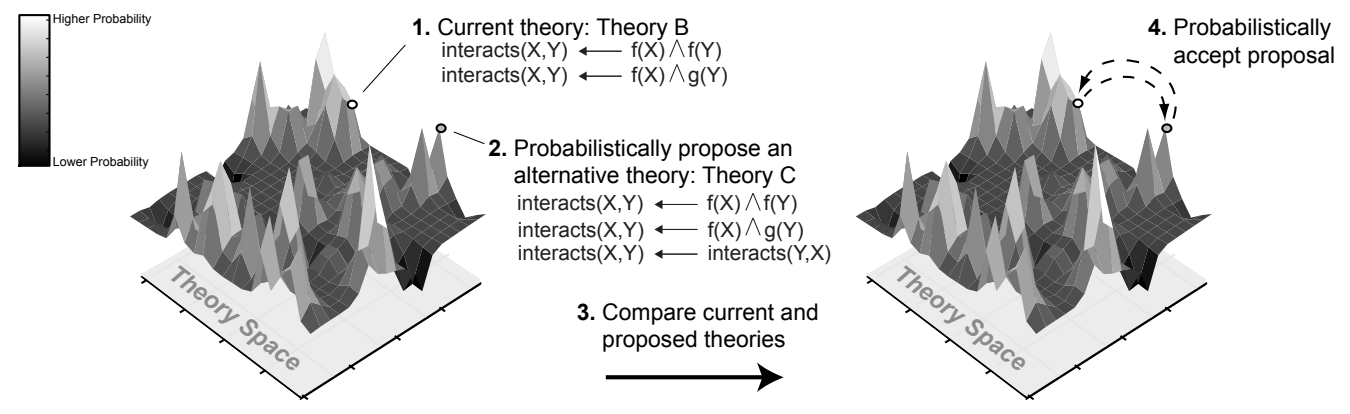

Figure 3: Schematic representation of the learning landscape within the domain of simple magnetism. Steps 1-4 illustrate the algorithmic process in this framework. The actual space of of theories is discrete, multidimensional and not necessarily locally connected.

At the level of computational theory, we can imagine an ideal Bayesian learner who computes the full posterior probability distribution over all possible theories, that is, who grasps this entire landscape and assesses its height at all points in parallel, conditioned on any given observed data set. But this is clearly unrealistic as a starting point for algorithmic accounts of children's learning, or any practical learning system with limited processing resources. Intuition suggests that children may simultaneously consider no more than a handful of candidate theories in their active thought, and developmentalists typically speak of the child's current theory as if, as in connectionist models, the learner's knowledge state corresponds to just a single point on the landscape rather than the whole surface or posterior distribution. The ideal Bayesian learner is in a sense similar to a person who has "not toiled 
but found" from the opening: the entire hypothesis space is already defined and the learner's task is merely to reshuffle probability over that space in response to evidence. The actual child must toil and construct her abstract theory, piece by piece, generalizing from experience.

Considering how a learner could move around on this landscape in search of the best theory, we see that most of the appealing properties of connectionist knowledge landscapes - the features that support efficient learning algorithms - are not present here. The geometry of the landscape is far from smooth: A small change in one of the concepts or laws of a theory will often lead to a drastic rise or fall in its plausibility, leading to a proliferation of isolated local maxima. There is typically no local information (such as a gradient) diagnostic of the most valuable directions in which to move. The landscape is even more irregular in ways that are not easily visualized. There is no uniform topology or neighborhood structure: the number and nature of variants that can be proposed by making local changes to the learner's current hypothesis vary greatly over the space, depending on the form of that hypothesis. Often changing one aspect of a theory requires others to be changed simultaneously in order to preserve coherence: for instance, if we posit a new abstract concept in our theory, such as the notion of a magnet, or if we remove a conceptual distinction (such as the distinction between magnets and magnetic objects), then one or more laws of the theory will need to be added, removed or redefined at the same time.

Artificial intelligence has a long history of treating learning in terms of search through a discrete space of symbolic descriptions, and a wide variety of search algorithms have been proposed to solve problems such as rule discovery, concept learning and generalization, scientific law discovery, and causal learning (Newell \& Simon, 1976; Mitchell, 1982; Bradshaw et al., 1983; Pearl, 2000; Spirtes et al., 2001). For some of these problems, there exist systematic search algorithms that can be as fast and reliable as the gradient-based optimization methods used in connectionist learning(Mitchell, 1982; Pearl, 2000; Spirtes et al., 2001). But for problems like scientific discovery (Bradshaw et al., 1983), or our formulation of children's theory learning, the best known search algorithms are not like this. Much like child learners, we suggest, these algorithms are slow, unreliable, and unsystematic (indeed often random), but with enough patience they can be expected to converge on veridical theories.

The specific search algorithm we describe is based on widely used methods in statistics and AI for approximating intractable Bayesian inferences, known as Markov Chain Monte Carlo (MCMC). MCMC algorithms have recently 
been proposed as models for the short-timescale dynamics of perceptual inferences in the brain (Gershman et al., 2009; Sundareswara \& Schrater, 2008; Moreno-Bote et al., 2011), but they are also well-suited to understanding the much longer-term dynamics of learning.

The remainder of this section sketches how our MCMC algorithm answers the two main challenges we set out at the start of this paper: explaining how children can reliably converge on veridical theories, given their constrained cognitive resources and a learning dynamic that often appears more random than systematic, and explaining how children can solve the hard "chickenand-egg" inference problem of jointly learning new concepts and new laws defined in terms of those concepts.

The heart of MCMC theory learning is an iterative loop of several basic steps, shown in Figure 3. The learner begins at some point in the theory landscape (e.g. theory B or C in Figure 3). The learner then proposes a possible move to a different theory, based on modifying the current theory's form: adding/deleting a law or set of laws, changing parts of a law or introducing a new concept, and so on. The proposed and current theories are compared based on evaluating (approximately) how well they explain the observed data (i.e., comparing the relative heights of these two points on the theory landscape). If the proposed theory scores higher, the learner accepts it and moves to this new location. If the proposal scores lower, the learner may still accept it or reject it (staying at the same location), with probability proportional to the relative scores of the two theories. These steps are then repeated with a new proposal based on the new current location.

From the standpoint of MCMC, randomness is not a problem but rather an essential tool for exploring the theory landscape. Because MCMC algorithms consider only one hypothesis at a time and propose local modifications to it, and there are no generally available signals (analogous to the error gradient in connectionist learning) for how to choose the best modification of the current hypothesis out of an infinite number of possible variations, the best learners can do is to propose variant theories to explore chosen in a randomized but hopefully intelligent fashion. Our algorithm proposes variants to the current hypothesis by replacing a randomly chosen part of the theory with another random draw from the probabilistic generative grammar for theories (that is, the prior over theories). This process could in principle propose any theory as a variant on any other, but it is naturally biased towards candidates that are most similar to the current hypothesis, as well as those that are a priori simpler and more readily generated by the grammar's templates 
for coherent laws. The use of law templates is crucial in focusing the random proposal mechanism on the most promising candidates. Without templates, all of the laws proposed could still have been generated from a more general grammar, but they would be much less likely a priori; learners would end up wasting most of their computational effort considering simple but uesless candidate laws. The templates make it likely that any random proposal is at least a plausibly useful explanation, not just a syntactically well-formed expression in the language of thought.

The decision of whether to accept or reject a proposed theory change is also made in a randomized but intelligently biased fashion. If a proposed change improves the theory's account of the data, it is always accepted, but sometimes a change that makes the theory worse could also be accepted. This probabilistic acceptance rule helps keep the leaner from becoming trapped for too long in poor local maxima of the theory landscape (Gilks \& Spiegelhalter, 1996).

Although we use MCMC as a search algorithm, aiming to find the best theory, the algorithm's proper function is not to find a single optimal theory but rather to visit all theories with probability proportional to their posterior probability. We can interpolate between MCMC as a posterior inference technique and MCMC as a search algorithm by annealing - or starting with more stochastic (or noisy) search moves and "lowering the temperature", making the search more deterministic over time (Kirkpatrick et al., 1983; Spall, 2003). This greatly improves convergence to the true theory. Such an algorithm can begin with little or no knowledge of a domain and, given enough time and sufficient data, reliably converge on the correct theory or at least some approximation thereof, corresponding to a small set of abstract predicates and laws.

Annealing is also responsible for giving the MCMC search algorithm some of its psychologically plausible dynamics. It gives rise to an early hightemperature exploration period characterized by a large number of proposed theories, most of which are far from veridical. As we see in young children, new theories are quick to be adopted and just as quick to be discarded. As the temperature is decreased, partially correct theories become more entrenched, it becomes rarer for learners to propose and accept large changes to their theories, and the variance between different theory learners goes down. As with older children, rational learners at the later stages of an annealed MCMC search tend to mostly agree on what is right, even if their theories are not perfect. Without annealing, MCMC dynamics at a constant temperature 
could result in a learner who is either too conservative (at low temperature) or too aggressive (at high temperature) in pursuing new hypotheses - that is, a learner who is prone to converge too early on a less-than-ideal theory, or to never converge at all.

Figures 6a and 7a illustrate these learning dynamics in action. (these are explained in detail in the next sections). On average, learners are consistently improving. On average, they are improving gradually. But individually, learners often get worse before they get better. Individually, they adopt theories in discrete jumps, signifying moments of fortuitous discovery. Such dynamics on the level of the individual learner are more in line with discovery processes in science and childhood than are the smoother dynamics of gradient descent on a typical connectionist energy landscape. Critics might reasonably complain that MCMC methods are slow and unreliable by comparison. But theory construction just is a difficult, time-consuming, painful and frustrating business - in both science and children's cognition. We can no more expect the dynamics of children's learning to follow the much tamer dynamics of gradient learning algorithms than we could expect to replace scientists with a gradient-based learning machine and see the discoveries of new concepts and new scientific laws emerging automatically. ${ }^{2}$ Currently we have no good alternative to symbolic representational machinery for capturing intuitive theories, and no good alternative to stochastic search algorithms for finding good points in the landscape of these symbolic theories.

What of the "hard problem of theory learning", the challenge of jointly learning new laws and new concepts defined only in terms of each other? Our MCMC search unfolds in parallel over two levels of abstraction: an outer loop in the space of theories, defined by sets of abstract laws; and an inner loop in the space of explanations or models generated by the theory for a particular domain of data, defined by groundings of the theory's concepts

\footnotetext{
${ }^{2}$ It is worth noting that not all connectionist architectures and learning procedures are confined to gradient-based methods operating on fixed parametric architectures. In particular the constructivist neural networks explored by Tom Shultz and colleagues Shultz (2003) are motivated by some of the same considerations that we are, aiming to capture the dynamics of children's discovery with learning rules that implement a kind of exploratory search. These models are still limited in their representational power, however: they can only express knowledge whose form and content fits into the connections of a neural network, and not the abstract concepts and laws that constitute an intuitive theory. For that reason we favor the more explicitly symbolic approach described here.
} 
on the specific entities of the domain. This two-level search lets us address the "chicken and egg" challenge by first proposing new laws or changes to existing laws of a theory in the outer search loop; these new laws can posit novel but 'blank' concepts of a certain form, whose meaning is then filled in the most plausible way on the next inner search loop. For example, the algorithm may posit a new rule never before considered, that objects of type $f$ interact with objects type $g$, without yet specifying what these concepts mean; they are just represented with blank predicates $f(X)$ and $g(X)$. The inner loop would then search for a reasonable assignment of objects to these classes - values for $f(X)$ and $g(X)$, for each object X - grounding them out as magnets and magnetic objects, for example. If this law proves useful in explaining the learner's observations, it is likely to persist in the MCMC dynamics, and with it, the novel concepts that began as blank symbols $f$ and $g$ but have now effectively become what we call "magnets" and "magnetic objects".

In sum, we see many reasons to think that stochastic search in a language of thought, with candidate theories generated by a probabilistic generative grammar and scored against observations in a hierarchical Bayesian framework, provides a better account of children's theory acquisition than alternative computational paradigms for modeling development such as connectionism. Yet there are also major gaps: scientists and young children alike are smarter, more active, more deliberate and driven explorers of both their theories and their experiences and experiments than are our MCMC algorithms (Schulz, 2012). We now turn to a more technical treatment of our model but we return to these gaps in the general discussion below.

\section{Formal framework}

This section gives a more formal treatment of theory learning, beginning with our hierarchical Bayesian framework for describing 'what' is learned (Figure 1), and then moving to our proposed MCMC algorithm for explaining 'how' it could be learned (Figure 3).

Formally, the hierarchical picture of knowledge shown in Figure 1 provides the backbone for a multilevel probabilistic generative model: conditional probability distributions that link knowledge at different levels of abstraction, supporting inference at any level(s) conditioned on knowledge or observations at other levels. For instance, given a domain theory $T$ and a set of noisy, sparse observations $D$, a learner can infer the most likely model $M$ and use 
that knowledge to predict other facts not yet directly observed (Katz et al., 2008; Kemp et al., 2008a). The theory $T$ sets the hypothesis space and priors for the model $M$, while the data $D$ determine the model's likelihood, and Bayes' rule combines these two factors into a model's posterior probability score,

$$
P(M \mid D, T) \propto P(D \mid M) P(M \mid T) .
$$

If the theory $T$ is unknown, the learner considers a hypothesis space of candidate theories generated by the higher-level universal theory $(U)$ grammar. $U$ defines a prior distribution over the space of possible theories, $P(T \mid U)$, and again the data $D$ determine a likelihood function, with Bayes' rule assigning a posterior probability score to each theory,

$$
P(T \mid D, U) \propto P(D \mid T) P(T \mid U) .
$$

Bayes' rule here captures the intuition of Occam's razor, that the theory which best explains a data set (has highest posterior probability $P(T \mid D, U)$ ) should balance between fitting the data well (as measured by the likelihood $P(D \mid T)$ ), and being simple or short to describe in our general language of thought (as measured by the prior $P(T \mid U)$ ). Probabilistic inference can operate in parallel across this hierarchical framework, propagating data-driven information upward and theory-based constraints downward to make optimal probabilistic inferences at all levels simultaneously.

Below we explain how each of these probability distributions is defined and computed. The first step is to be more precise about how we represent theories, which we have described so far using an informal mix of logic and natural langauge but now formalize using first-order predicate logic.

A language for theories. Following (Katz et al., 2008) we choose to represent the laws in a theory as Horn clauses, logical expressions of the form $r \leftarrow(f \wedge g \wedge \ldots \wedge s \wedge t)$, where each term $r, f, g, s, t, \ldots$ is a predicate expressing an attribute or relation on entities in the domain, such as $f(X)$ or $s(X, Y)$. Horn clauses express logical implications - a set of conjunctive conditions under which $r$ holds - but can also capture intuitive causal relations (Kemp et al., 2007) under the assumption that any propositions not generated by the theory are assumed to be false. The use of implicational clauses as a language for causal theories was explored extensively in (Feldman, 2006).

While richer logical forms are possible, Horn clauses provide a convenient and tractable substrate for exploring the ideas of stochastic search over a space of theories. In our formulation, the Horn clauses contain two kinds of 
predicates: "core" and "surface". Core predicates are those that cannot be reduced further using the theory's laws. Surface predicates are derived from other predicates, either surface or core, via the laws. Predicates may or may not be directly observable in the data. The core predicates can be seen as compressing the full model into just the minimal bits necessary to specify all true facts. As we explain in more detail below, a good theory is one that compresses a domain well, that explains as much of the observed data as possible using only the information specified in the core predicates. In our magnetism example, the core could be expressed in terms of two predicates $f(X)$ and $g(X)$. Based on an assignment of truth values to these core predicates, the learner can use the theory's laws such as interacts $(X, Y) \leftarrow f(X) \wedge g(Y)$ to derive values for the observable surface predicate interacts $(X, Y)$. For $n$ objects, there are $O\left(n^{2}\right)$ interactions that can be observed (between all pairs of objects) but these can be explained and predicted by specifying only $O(n)$ core predicate values (for each object, whether or not it is a magnet or is magnetic).

As another example of how a theory supports compression via its core predicates and abstract laws, consider the domain of kinship as shown in Figure 1. A child learning this domain might capture it by core predicates such as parent, spouse, and gender, and laws such as "Each child has two parents of opposite gender, and those parents are each others' spouse"; "A male parent is a father"; "Two individuals with the same parent are siblings"; "A female sibling is a sister"; and so on. Systems in this domain would correspond to individual families that the child knows about. A system could then be compressed by specifying only the values of the core predicates, for example which members of a family are spouses, who is the parent of whom, and who is male or female. From this minimal set of facts and concepts all other true facts about a particular family can be derived, predicting new relationships that were not directly observed.

In constructing a theory, the learner introduces abstract predicates via new laws, or new roles in existing laws, and thereby essentially creates new concepts. Notice that the core predicates in our magnetism theory need be represented only in purely abstract terms, $f(X)$ and $g(X)$, and initially they have only this bare abstract meaning. They acquire their meaning as concepts picking out magnets or magnetic objects respectively in virtue of the role they play in the theory's laws and the explanations they come to support for the observed data. This is the sense in which our framework allows the introduction of genuinely new abstract concepts via their inferential or 
conceptual roles.

Entities may be typed and predicates restricted based on type constraints. For example, in the taxonomy theory shown in Figure 1, has_a $(X, Y)$ requires that $\mathrm{X}$ be a category and $\mathrm{Y}$ be a property, while $i s_{-} a(X, Y)$ requires that $\mathrm{X}$ and $\mathrm{Y}$ both be categories. Forcing candidate models and theories to respect these type constraints provides the learner with another valuable and cognitively natural inductive bias.

Although our focus here is on the acquisition of intuitive theories in general, across all domains of knowledge and all ages, much research has been concerned with the form of young children's theories in a few core domains and the development of that knowledge over the first years of life. Our hornclause language is too limited to express the full richness of a two-year-old's intuitive physics or intuitive psychology, but it can represent simplified versions of them. For example, in Figure 1 we show a fragment of a simple "desire psychology" theory, one hypothesized early stage in the development of intuitive psychology around two years of age (Wellman \& Woolley, 1990). This theory aims to explain agents' goal-directed actions, such as reaching for, moving towards or looking for various object, in terms of basic but unobservable desires. In our language desires $(X, Y)$ (or simply $d(X, Y)$ in Figure 1) is a core predicate relating an agent $X$ to an object $Y$. Desires are posited to explain observations of a surface predicate goes_to $(X, Z, S)$ : agent $X$ goes to (or reaches for or looks in) location $Z$ in situation $S$. We also introduce background information in the form of an additional predicate location $(Y, Z, S)$ available to the child, specifying that object $Y$ is in location $Z$ in situation $S$. Then by positing which agents desire which objects, and a law that says effectively, "an agent will go to a certain location in a given situation if that location contains an object that the agent desires", a child can predict how agents will act in various situations, and explain why they do so.

The theory prior $P(T \mid U)$. We posit $U$ knowledge in the form of a probabilistic context-free Horn clause grammar (PHCG) that generates the hypothesis space of possible Horn-clause theories, and a prior $P(T \mid U)$ over this space (Figure 4). This grammar and the Monte Carlo algorithms we use to sample or search over the theory posterior $P(T \mid D, U)$ are based heavily on (Goodman et al., 2008b), who introduced the approach for learning single 
Top level theory

$$
\begin{array}{ll}
\mathrm{S} & \Rightarrow \quad(\text { Law }) \wedge \mathrm{S} \\
\mathrm{S} & \Rightarrow(\text { Tem }) \wedge \mathrm{S} \\
\mathrm{S} & \Rightarrow \text { Stop }
\end{array}
$$

Random law generation

(Law)

(Add1)

(Add2)

\section{Predicate generation}

$$
\left(\mathrm{F}_{\text {left }} 1\right)
$$$$
\vdots
$$$$
\left(\mathrm{F}_{\text {left }} \alpha\right)
$$$$
\left(\mathrm{F}_{\text {right }} 1\right)
$$

Law $\Rightarrow \quad\left(\mathrm{F}_{\text {left }} \leftarrow \mathrm{F}_{\text {right }} \wedge\right.$ Add $)$

$\mathrm{A} \quad \Rightarrow \mathrm{F} \wedge$ Add

A $\quad \Rightarrow$ Stop

$$
\vdots
$$$$
\left(\mathrm{F}_{\text {right }} \alpha\right)
$$$$
\left(\mathrm{F}_{\text {right }}(\alpha+1)\right)
$$$$
\text { : }
$$$$
\left(\mathrm{F}_{\text {right }}(\alpha+\beta)\right)
$$

$$
\begin{aligned}
& \mathrm{F}_{\text {left }} \Rightarrow \text { surface } 1() \\
& \mathrm{F}_{\text {left }} \Rightarrow \text { surface } \alpha() \\
& \mathrm{F}_{\text {right }} \Rightarrow \text { surface } 1()
\end{aligned}
$$$$
\mathrm{F}_{\text {right }} \Rightarrow \text { surface } \alpha()
$$$$
\mathrm{F}_{\text {right }} \Rightarrow \text { core } 1()
$$$$
\mathrm{F}_{\text {right }} \Rightarrow \operatorname{core} \beta()
$$

Law templates

$\begin{array}{lll}(\text { Tem } 1) & \text { Tem } & \Rightarrow \text { template } 1() \\ \vdots & & \\ (\text { Tem } \gamma) & \text { Tem } \Rightarrow \text { template } \gamma()\end{array}$

Figure 4: Production rules of the Probabilistic Horn Clause Grammar. $S$ is the start symbol and Law, Add, F and Tem are non-terminals. $\alpha, \beta$, and $\gamma$ are the numbers of surface predicates, core predicates, and law templates, respectively. 
rule-based concepts rather than the larger law-based theory structures we consider here. We refer readers to (Goodman et al., 2008b) for many technical details. Given a set of possible predicates in the domain, the PHCG draws laws from a random construction process (Law) or from law templates (Tem; explained in detail below) until the Stop symbol is reached, and then grounds out these laws as horn clauses. The prior $P(T \mid U)$ is the product of the probabilities of choices made at each point in this derivation. Because all these probabilities are less than one, the prior favors simpler theories with shorter derivations. The precise probabilities of different laws in the grammar are treated as latent variables and integrated out, which favors re-use of the same predicates and law components within a theory (Goodman et al., 2008b).

Law templates. We make the grammar more likely to generate useful laws by equipping it with templates, or canonical forms of laws that capture structure likely to be shared across many domains. While it is possible for the PHCG to reach each of these law forms without the use of templates, their inclusion allows the most useful laws to be invented more readily. They can also serve as the basis for transfer learning across domains. For instance, instead of having to re-invent transitivity anew in every domain with some specific transitive predicates, a learner could recognize that the same transitivity template applies in several domains. It may be costly to invent transitivity for the first time, but once found - and appreciated! - its abstract form can be readily re-used. The specific law templates used are described in Figure 5. Each " $F(\cdot)$ " symbol stands for a non-terminal representing a predicate of a certain -arity. This non-terminal is later instantiated by a specific predicate. For example, the template $F(X, Y) \leftarrow F(X, Z) \wedge F(Z, Y)$ might be instantiated as $i s \_a(X, Y) \leftarrow i s \_a(X, Z) \wedge i s_{\_} a(Z, Y)$ (a familiar transitive law) or as has_a $(X, Y) \leftarrow i s_{-} a(X, Z) \wedge h a s_{\_} a(Z, Y)$ (the other key law of taxonomy, which is like saying that "has_a is transitive over $i s_{\_} a$ "). This template could be instantiated differently in other domains, for example in kinship as $\operatorname{child}(X, Y) \leftarrow \operatorname{child}(X, Z) \wedge \operatorname{spouse}(Z, Y)$, which states that the child-parent relationship is transitive over spouse.

The theory likelihood $P(D \mid T)$. An abstract theory makes predictions about the observed data in a domain only indirectly, via the models it generates. A theory typically generates many possible models: even if a child 


$$
\begin{array}{l|rl}
\mathrm{F}(\mathrm{X}, \mathrm{Y}) \leftarrow \mathrm{F}(\mathrm{X}, \mathrm{Z}) \wedge \mathrm{F}(\mathrm{Z}, \mathrm{Y}) & \mathrm{F}(\mathrm{X}, \mathrm{Y}) & \leftarrow \mathrm{F}(\mathrm{X}) \wedge \mathrm{F}(\mathrm{Y}) \\
\mathrm{F}(\mathrm{X}, \mathrm{Y}) \leftarrow \mathrm{F}(\mathrm{Z}, \mathrm{X}) \wedge \mathrm{F}(\mathrm{Z}, \mathrm{Y}) & \mathrm{F}(\mathrm{X}, \mathrm{Y}) \leftarrow \mathrm{F}(\mathrm{Y}, \mathrm{X}) \\
\mathrm{F}(\mathrm{X}, \mathrm{Y}) \leftarrow \mathrm{F}(\mathrm{X}, \mathrm{Z}) \wedge \mathrm{F}(\mathrm{Y}, \mathrm{Z}) & \mathrm{F}(\mathrm{X}, \mathrm{Y}) \leftarrow \mathrm{F}(\mathrm{X}, \mathrm{Y}) \\
\mathrm{F}(\mathrm{X}, \mathrm{Y}) \leftarrow \mathrm{F}(\mathrm{Z}, \mathrm{X}) \wedge \mathrm{F}(\mathrm{Y}, \mathrm{Z}) & \mathrm{F}(\mathrm{X}) & \leftarrow \mathrm{F}(\mathrm{X}) \\
\mathrm{F}(\mathrm{X}, \mathrm{Y}) \leftarrow \mathrm{F}(\mathrm{X}, \mathrm{Y}) \wedge \mathrm{F}(\mathrm{X}) & \mathrm{F}(\mathrm{X}) & \leftarrow \mathrm{F}(\mathrm{X}, \mathrm{Y}) \wedge \mathrm{F}(\mathrm{X}) \\
\mathrm{F}(\mathrm{X}, \mathrm{Y}) \leftarrow \mathrm{F}(\mathrm{Y}, \mathrm{X}) \wedge \mathrm{F}(\mathrm{X}) & \mathrm{F}(\mathrm{X}) & \leftarrow \mathrm{F}(\mathrm{Y}, \mathrm{X}) \wedge \mathrm{F}(\mathrm{X}) \\
\mathrm{F}(\mathrm{X}, \mathrm{Y}) \leftarrow \mathrm{F}(\mathrm{X}, \mathrm{Y}) \wedge \mathrm{F}(\mathrm{Y}) & \mathrm{F}(\mathrm{X}) & \leftarrow \mathrm{F}(\mathrm{X}, \mathrm{Y}) \wedge \mathrm{F}(\mathrm{Y}) \\
\mathrm{F}(\mathrm{X}, \mathrm{Y}) \leftarrow \mathrm{F}(\mathrm{Y}, \mathrm{X}) \wedge \mathrm{F}(\mathrm{Y}) & \mathrm{F}(\mathrm{X}) & \leftarrow \mathrm{F}(\mathrm{Y}, \mathrm{X}) \wedge \mathrm{F}(\mathrm{Y})
\end{array}
$$

Figure 5: Possible templates for new laws introduced by the grammar. The leftmost $F$ can be any surface predicate, the right $F$ can be filled in by any surface or core predicates, and $X$ and $Y$ follow the type constraints.

has the correct theory and abstract concepts of magnetism, she could categorize a specific set of metal bars in many different ways, each of which would predict different interactions that could be observed as data. Expanding the theory likelihood,

$$
P(D \mid T)=\sum_{M} P(D \mid M) P(M \mid T),
$$

we see that theory $T$ predicts data $D$ well if it assigns high prior $P(M \mid T)$ to models $M$ that make the data probable under the observation process $P(D \mid M)$.

The model prior $P(M \mid T)$ reflects the intuition that a theory $T$ explains some data well if it compresses well: if it requires few additional degrees of freedom beyond its abstract concepts and laws - that is, few specific and contingent facts about the system under observation, besides the theory's general prescriptions - to make its predictions. This intuition is captured by a prior that encourages the core predicates to be as sparse as possible, thereby penalizing theories that can only fit well by "overfitting" with many extra degrees of freedom. This sparseness assumption is reasonable as a starting point for many domains, given that core predicates are meant to explain and 
compress the data. Formally, we assume a conjugate beta prior on all binary facts in $M$, modeled as Bernoulli random variables which we integrate out analytically, as in (Katz et al., 2008).

Finally, the model likelihood $P(D \mid M, T)$ comes from assuming that we are observing randomly sampled true facts (sampled with replacement, so the same fact could be observed on multiple occasions), which also encourages the model extension to be as small as possible. This provides a form of implicit negative evidence (Tenenbaum \& Griffiths, 2001), useful as an inductive bias when only positive facts of a domain are observed.

Stochastic search in theory space: a grammar-based Monte Carlo algorithm. Following (Goodman et al., 2008b), we use a grammarbased Metropolis-Hastings (MH) algorithm to sample theories from the posterior distribution over theories conditioned on data, $P(T \mid D, U)$. This algorithm is applicable to any grammatically structured theory space, such as the one generated by our PHCG; it is also a version of the Church MH inference algorithm (Goodman et al., 2008a). The MH algorithm is essentially a Markov chain on the space of potential derivations from the grammar, where each step in the chain - each proposed change to the current theory - corresponds to regenerating some subtree of the derivation tree from the PHCG. For example, if our theory of magnetism includes the law interacts $(X, Y) \leftarrow f(X) \wedge g(Y)$, the $\mathrm{MH}$ procedure might propose to add or delete a predicate (e.g., interacts $(X, Y) \leftarrow f(X) \wedge g(Y) \wedge h(Y)$ or

interacts $(X, Y) \leftarrow f(X))$, to change one predicate to an alternative of the same form (e.g., interacts $(X, Y) \leftarrow f(X) \wedge h(Y))$ or a different form if available (e.g., interacts $(X, Y) \leftarrow f(X) \wedge s(X, Y))$; to resample the law from a template (e.g., interacts $(X, Y) \leftarrow t(X, Z) \wedge t(Z, Y))$; or to add or delete a whole law.

These proposals are accepted with probability equal to the maximum of 1 and the $\mathrm{MH}$ acceptance ratio,

$$
\frac{P\left(T^{\prime} \mid D, U\right)}{P(T \mid D, U)} \cdot \frac{Q\left(T \mid T^{\prime}\right)}{Q\left(T^{\prime} \mid T\right)},
$$

where $T$ is the current theory, $T^{\prime}$ is the new proposed theory, and $Q(\cdot \mid \cdot)$ is the transition probability from one theory to the other, derived from the PHCG (Goodman et al., 2008b). To aid convergence we raise these acceptance ratios to a power greater than 1, which we increase very slightly after each $\mathrm{MH}$ step in a form of simulated annealing. Early on in learning, a learner is thus more likely to try out a new theory that appears worse than 
the current one, exploring candidate theories relatively freely. However, with time the learner becomes more conservative - increasingly likely to reject new theories unless they lead to an improved posterior probability.

While this $\mathrm{MH}$ algorithm could be viewed merely as a way to approximate the calculations necessary for a hierarchical Bayesian analysis, we suggest that it could also capture in a schematic form the dynamic processes of theory acquisition and change in young children. Stochastic proposals to add a new law or change a predicate within an existing law are consistent with some previous characterizations of children's theory learning dynamics (Siegler \& Chen, 1998). These dynamics were previously proposed on purely descriptive grounds, but here they emerge as a consequence of a rational learning algorithm. Although the dynamics of an $\mathrm{MH}$ search might appear too random to an omniscient observer who knows the "true" target of learning, it would not be fair to call the algorithm sub-optimal, because it is the only known general-purpose approach for effectively searching a complex space of logical theories. Likewise, the annealing process that leads learning to look child-like in a certain sense - starting off with more variable, rapidly changing and adventurous theories, then becoming more conservative and less variable over time - also makes very good engineering sense. Annealing has proven to be useful in stochastic search problems across many scientific domains (Kirkpatrick et al., 1983) and is the only known method to ensure that a stochastic search converges to the globally optimal solution. It does not seem implausible that some cognitive analog of annealing could be at work in children's learning. ${ }^{3}$

Approximating the theory score: an inner loop of MCMC Computing the theory likelihood $P(D \mid T)$, necessary to compare alternative theories in Equation (4), requires a summation over all possible models consistent with the current theory (Equation (3)). Because this sum is typically very hard to evaluate exactly, we approximate $P(D \mid T)$ with $P\left(D \mid M^{*}, T\right) P\left(M^{*} \mid T\right)$, where $M^{*}$ is an estimate of the maximum a-posteriori (MAP) model in-

\footnotetext{
${ }^{3}$ It is worth noting that annealing could be implemented in a learning system without an explicit temperature parameter or cooling schedule, merely based on experience accumulating over time. Here for simplicity we have kept the learner's dataset fixed, but if the learner is exposed to increasing amounts of data over time and treats all data as independent samples from the model, this also acts to lower the effective temperature by creating larger ratios between likelihoods (and hence posterior probabilities) for a given pair of theories.
} 
ferred from the data: the most likely values of the core predicates. The MAP estimate $\mathrm{M}^{*}$ is obtained by running an inner sampling procedure over the values of the core predicates. As in (Katz et al., 2008), we use a specialized form of Metropolis-Hastings sampling known as Gibbs sampling. The Gibbs sampler goes over each core predicate assignment in turn while keeping all other assignments fixed, and proposes changes to the currently considered assignment. As a concrete example of how the Gibbs loop works, consider a learner who is proposing a theory that contains the law interacts $(X, Y) \leftarrow f(X) \wedge g(Y)$, i.e., objects for which core predicate $f$ is true interact with objects for which core predicate $g$ is true. The learner begins by randomly extending the core categories over the domain's objects: e.g., $f$ might be posited to hold for objects 1,4 , and 7 , while $g$ holds for objects 2, 4, 6, and 8. (Note how either, both or none of the predicates may hold for any object, a priori.) The learner then considers the extension of predicate $f$ and proposes removing object 1 , scoring the new model (with all other assignments as before) on the observed data and accepting the proposed change probabilistically depending on the relative scores. The learner then considers objects 2,3 , and so on in turn, considering for each object whether predicate $f$ should apply, before moving on to predicate $g$. (These object-predicate pairs are often best considered in random order on each sweep through the domain.) This process continues until a convergence criteria is reached. We anneal slightly on each Gibbs sweep to speed convergence and lock in the best solution. The Gibbs sampler over models generated by a given theory is thus an "inner loop" of sampling in our learning algorithm, operating within each step of an "outer loop" sampling at a higher level of abstract knowledge, the MH sampler over theories generated by $U$ knowledge.

\section{Case Studies}

We now explore the performance of this stochastic approach to theory learning in two case studies, using simulated data from the domains of taxonomy and magnetism introduced above. We examine the learning dynamics in each domain and make more explicit the possible parallels with human theory acquisition. 


\subsection{Taxonomy}

As we saw earlier, the domain of taxonomy illustrates how a compressive knowledge representation is useful in capturing semantic data. How can such a powerful organizing principle itself be learned? Katz et al. (2008) showed that a Bayesian ideal observer can pick out the best theory of taxonomy given a small set of eight possible alternatives. Here we show that the theory of taxonomy can be learned in a more constructive way, via an MCMC search through our infinite grammar-generated hypothesis space. The theory to be learned takes the following form:

Two core predicates: $\quad s(X, Y)$ and $t(X, Y)$

Two observable predicates: $\quad i s \_a(X, Y)$ and has_a $(X, Y)$

$\begin{array}{rrrl}\text { Law 1: } & & i s_{\_} a(X, Y) & \leftarrow s(X, Y) \\ \text { Law 2: } & & \text { has_a }(X, Y) & \leftarrow t(X, Y) \\ \text { Law 3: } & i s_{\_} a(X, Y) & \leftarrow i s_{\_} a(X, Z) \wedge i s_{\_} a(Z, Y) \\ \text { Law 4: } & & \text { has_a }(X, Y) & \leftarrow i s_{\_} a(X, Z) \wedge h a s \_a(Z, Y)\end{array}$

These laws by themselves do not yet capture the complete knowledge representation we are after; we also need to instantiate the core predicates in a particular model. These laws allow many possible models for any given data sets. One of these models is the compressed tree representation (shown in Figure 1 in the Model section of the taxonomy domain), which specifies only the minimal facts needed to derive the observed data from Laws 1-4. A different model could link explicitly all the $i s \_a(X, Y)$ connections, for example drawing the links between salmon and animal, shark and animal and so on. Another model could link explicitly all the has_a $(X, Y)$ connections. However, these latter two models would be much less sparse than the compressed tree representation, and thus would be disfavored relative to the compressed tree shown in Figure 1, given how we have defined the model prior $P(M \mid T)$. In sum, in this framework, the organization of categories and properties into a tree-structured inheritance hierarchy comes about from a combination of positing the appropriate abstract laws and core predicates together with a sparsity preference on the assignments of the core predicates' values.

Note also that the core predicates $s(X, Y)$ and $t(X, Y)$ acquire their meaning in part by their inferred extensions, and in part by how they are related 
to the observed surface predicates. The surface predicates are assumed to be verbal labels which the learner observes and needs to account for. The link between these verbal labels and the core relations are what given by Laws 1 and 2. While these links could in general also be learned, we follow Katz et al. (2008) in taking Laws 1 and 2 as given for this particular domain and asking whether a learner can theoretically discover Laws 3 and 4 -but now at the algorithmic level. We test learning for the same simple model of the

a.

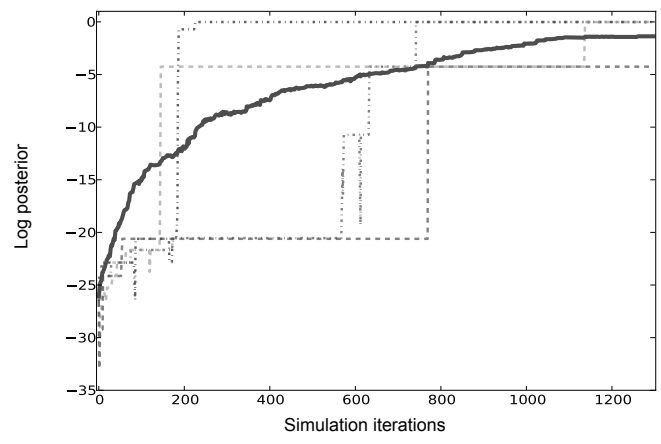

b.

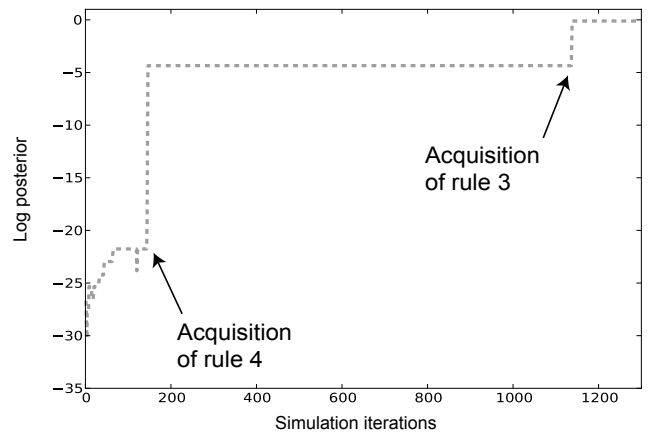

Figure 6: Representative runs of theory learning in Taxonomy. (a) Dashed lines show different runs. Solid line is the average across all runs. (b) Highlighting a particular run, showing the acquisition of law 4, followed by the acquisition of law 3 and thus achieving the final correct theory.

taxonomy domain studied by Katz et al., using seven categories and seven properties in a balanced tree structure. We presented all true facts from this model as observations to the learner, including both property statements (e.g., "An eagle has claws") and category membership statements (e.g., "An eagle is a bird"). The data for this section and the following case study can be found in the appendix.

We ran 60 simulations, each comprising 1300 iterations of the outer $\mathrm{MH}$ loop (i.e., moves in the space of theories). Four representative runs are shown in Figure 6, as well as the average across all the runs. Out of 60 simulations, 52 found the correct theory within the given number of iterations, and 8 discovered a partial theory which included only Law 3 or Law 4.

Several points are worth noting beyond these quantitative results. First, it is striking that abstract structure can be learned effectively from very little data. Using simple local search, our learning algorithm is able to navigate an infinite space of potential theories and discover the true laws underlying 
the domain, even with relatively few observations in the relations between seven categories and seven properties. This is a version of the "blessing of abstraction" described in Goodman et al. (2011) and Tenenbaum et al. (2011), but one that is realized at the algorithmic level and not just the computational level of ideal learning.

Second, individual learning trajectories proceed in a characteristic pattern of stochastic leaps. Discovering the right laws gives the learner strong explanatory power. However, surrounding each "good" theory in the discrete hypothesis space are many syntactically similar but nonsensical or much less useless formulations. Moving from a good theory to a better one thus depends on proposing just the right changes to the current hypothesis. Since these changes are proposed randomly, the learner often stays with a particular theory for many iterations, rejecting many proposed alternatives which score worse or not significantly better than the current theory, until a new theory is proposed that is so much better it is almost surely accepted. This leads to the observed pattern of plateaus in the theory score, punctuated by sudden jumps upward and occasional jumps downward in probability. While we do not want to suggest that people learn theories only by making random changes to their mental structures, the probabilistic nature of proposals in a stochastic search algorithm could in part explain why individual human learning curves rarely proceed along a smooth path and can show broad variation across individuals given the same data.

Third, while individual learning trajectories may be discontinuous, on average learning appears smooth. Aggregating performance over all runs shows a smooth improvement of the theory's score that belies the underlying discrete nature of learning at an individual level. This emphasizes the possible danger of studying theory learning and theory change only in the average behavior of groups of subjects, and the theoretical value of microgenetic methods (Siegler \& Crowley, 1991) for constraining algorithmic-level models of children's' learning.

\subsection{Magnetism}

We now turn to the domain of magnetism, where the trajectory of theory learning reveals not only successful acquisition, but interesting intermediate stages and transitions corresponding to classic phenomena of conceptual change in childhood and early science (Carey, 2009). The simplified theory of magnetism to be learned takes the following form: 
Two core predicates: $\quad f(X)$ and $g(X)$

One observable predicate: $\quad$ interacts $(X, Y)$
Law 1:
$\operatorname{interacts}(X, Y) \leftarrow f(X) \wedge f(Y)$
Law 2:
interacts $(X, Y) \leftarrow f(X) \wedge g(Y)$
Law 3:
interacts $(X, Y) \leftarrow$ interacts $(X, Y)$

The particular model used for learning contained 10 objects: 3 magnets, 5 magnetic objects and 2 non-magnetic objects. The learner was given all true facts in this model, observing interactions between each magnet and every other object that was either a magnet or a magnetic object, but no other interactions. Unlike in the previous taxonomy example, the learner was given none of the laws or core predicate structure to begin with; the entire theory had to be constructed by the search algorithm. Assuming the correct laws (as shown above) can be found, the model prior $P(M \mid T)$ favoring sparsity suggests the optimal values for the core predicates should assign one core predicate $(f)$ to all and only the magnets, and another predicate to all and only the non-magnet magnetic objects. This leads to the theory and model depicted jointly in Figure 1.

a.

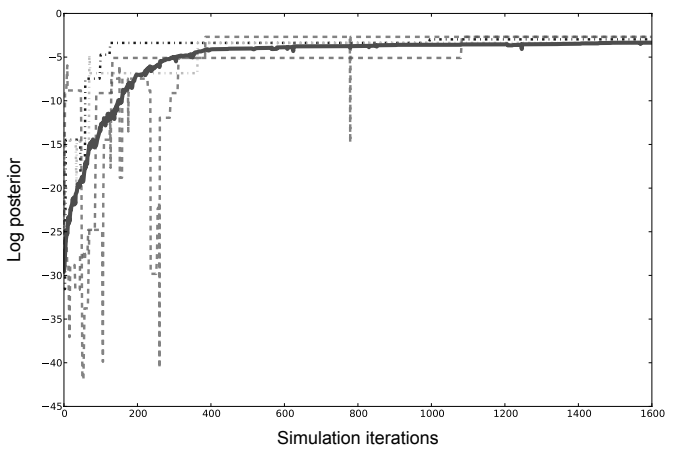

b.

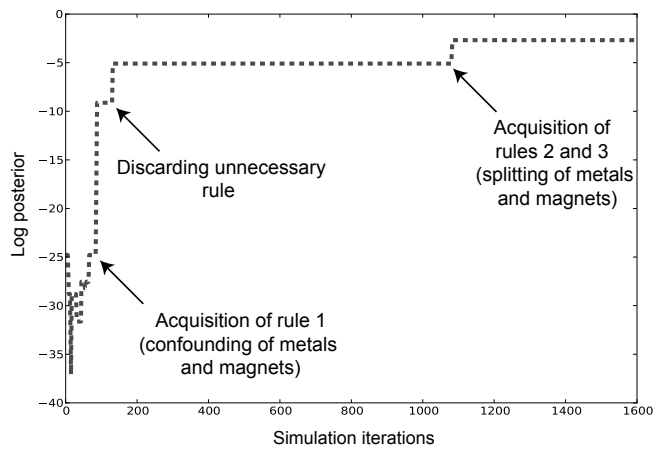

Figure 7: Representative runs of theory learning in Magnetism. (a) Dashed lines show different runs. Solid line is the average across all runs. (b) Highlighting a particular run, showing the acquisition of law 1 and the confounding of magnets and magnetic (but nonmagnet) objects, the discarding of an unnecessary law which improves the theory prior, and the acquisition of the final correct theory. 
We ran 70 simulations, each comprising 1600 iterations of the outer $\mathrm{MH}$ loop sampling over candidate theories. In many respects, results in this domain were qualitatively similar to what we described above for taxonomy. Out of 70 simulated learning runs, 50 found the correct theory or a minor logical variant of it; the rest discovered a partial theory. The correct final theories account for the full observed data and only the observed data, using three laws. While all the full theories learned included Laws 1 and 2, only some of them included the exact form of Law 3, expressing the symmetry of interaction. ${ }^{4}$ The dynamics of representative runs are displayed in Figure 7, as well as the average over all the runs. As in the domain of taxonomy, individual learners experienced radical jumps in their theories, while aggregating across runs learning appears to be much smoother.

The most interesting aspects of learning here were found in the transitions between distinct stages of learning, when novel core predicates are introduced and existing core predicates shift their meaning in response. Key transitions in children's cognitive development may be marked by restructuring of concepts, as when one core concept differentiates into two (Carey, 1985). Our learning algorithm often shows this same dynamic in the magnetism task. There is no single order of concept acquisition that the algorithm follows in all or most runs, but the most common trajectory (shown in Figure 7b) involves learning Law 1 first, followed later by the acquisition of Laws 2 and 3. As mentioned earlier, for a learner who knows only Law 1, the optimal setting of the core predicates is to lump together magnets and magnetic objects in one core predicate, essentially not differentiating between them. Only when Laws 2 and 3 are learned does the learner also acquire a second core predicate that carves off the magnetic non-magnets from the magnets. On a smaller number of runs, a different order of acquisition is observed: first Laws 2 and 3 are learned, and then Law 1 is added. This sequence also involves a conceptual restructuring, albeit a less dramatic one. A learner who

\footnotetext{
${ }^{4}$ However, the variants discovered were functionally equivalent within this domain to symmetry. Such variants include redundant re-statements of symmetry, such as inter$\operatorname{acts}(\mathrm{X}, \mathrm{Y}) \leftarrow \operatorname{interacts}(\mathrm{Y}, \mathrm{Z}) \wedge$ equals $(\mathrm{Z}, \mathrm{X})$. Other forms happen to capture the same facts as symmetry within this particular domain, such as interacts $(\mathrm{X}, \mathrm{Y}) \leftarrow \operatorname{interacts}(\mathrm{Y}, \mathrm{Z}) \wedge$ $\mathrm{g}(\mathrm{Z})$. These variants appear more complex than the basic symmetry law, and they do score slightly worse than theories that recover the original formulation. However, since they were generated by templates in this case, this extra complexity does not hurt them significantly.
} 
possesses only Laws 2 and 3 will optimally assign one predicate to all and only the magnets, and another core predicate to both magnets and magnetic non-magnets, again lumping these two classes together. Only once Law 1 is added to Laws 2 and 3 will the learner completely differentiate the two core predicates with non-overlapping extensions corresponding to magnets and magnetic non-magnets.

In both of these cases, the time course of learning appears as a progression from simpler theories (with fewer core predicates and/or laws) that explain the data less faithfully or less efficiently, to more complex theories (with more core predicates and/or laws) that explain the data more faithfully or more efficiently. A learner with the simpler theory consisting of only Law 1 (without Laws 2 and 3) will overgeneralize, predicting the existence of interactions that do not actually occur: interactions between pairs of nonmagnet magnetic objects (which would be treated the same as interactions between two magnets, or a magnet and a magnetic object). A learner with the simpler theory consisting of Laws 2 and 3 (but not Law 1) will make the right predictions about interactions to be observed but would represent the world less efficiently, less sparsely, than they could: they would need to assign values for both core predicates to represent each magnet, rather than just using a single core predicate to represent magnets and only magnets. Yet while being less accurate or less efficient, these earlier, simpler theories are still reasonable first approximations to the optimal theory of this domain. They are also plausible intermediate points for the learner on the way to the optimal theory, who can get there merely by adding one or two new laws and differentiating the extension of a core predicate into two non-overlapping subsets of objects, magnets and magnetic non-magnets, which had previously been merged together in that predicate's extension.

\section{Two Sources of Learning Dynamics}

The story of development is in essence one of time and data. In order to construct adult-level intuitive theories, children require both sufficient time to ponder and exposure to sufficient evidence. For a child on the verge of grasping a new theory, either additional data or additional time to think can make the difference (Carey, 2009). Measured as a function of either time or amount of data experienced, the dynamics of learning typically follows an arc from simpler theories that only coarsely predict or encode experience to more complex theories that more faithfully predict and encode it. 
The above case studies of theory learning in the domains of taxonomy and magnetism show this dynamic as a function of time elapsed in the search process, for a fixed data set. Previous Bayesian models of theory learning (Kemp \& Tenenbaum, 2009) have emphasized the complementary perspective: how increasing amounts of data naturally drive an ideal learner to more complex but more predictive theories, independent of the dynamics of search or inference.

These two sources of learning dynamics are most naturally understood at different levels of analysis. Data-driven learning dynamics seems best explained at the computational level, where the ideal learner shifts probability mass between candidate theories as a function of the data observed. In contrast, time-driven dynamics (independent of the amount of data observed) seems best approached at the algorithmic level, with models that emphasize how the learner's process of searching over a hypothesis space unfolds over time independent of the pace with which data accumulates.

Our modeling approach is well suited to studying both data-driven and time-driven dynamics and their interactions, because of its focus on the interface between the computational and algorithmic levels of analysis. In the rest of this section we return to the domain of simplified magnetism and explore the independent effects and interactions of these two different drivers of theory change in our model. How does varying time and data affect our ideal learner? We provide the learner with several different data sets, and examine how the learning dynamics unfold over time for each one of these sets. In each data set we provide the learner with different observations by parametrically varying the number of magnetic objects over five cases, which can be ordered in the following way: Case 1 had 3 magnets, 1 magnetic object and 6 non-magnetic objects. Each case then adds one magnetic object while removing one non-magnetic object, so that case 2 has 3 magnets, 2 magnetic objects and 5 non-magnetic objects, up to case 5 which has 3 magnets, 5 magnetic objects and 2 non-magnetic objects (the same as the previous section). We also considered a special case, case $\mathrm{X}$, in which there is only 1 magnet, 7 magnetic objects and 2 non-magnetic object. In all cases the theory governing the domain is exactly the same as that described in the magnetism case study. Given these different cases we find that at the end of the simulation the learner almost always settled on one of three theories. We therefore focus on these three theories, the formal laws of which are given in Figure 8a. Informally, these theories correspond to:

Theory A: "There is one class of interacting objects in the world, and 
objects in this class interact with other objects in this class."

Theory B: "There are two classes of interacting objects in the world, and objects from one class interact with objects in the other class. These interactions are symmetric."

Theory C: "There are two classes of interacting objects in the world, and objects from one class interact with objects in the other class. Also, objects in one of the classes interact with other objects in the same class. These interactions are symmetric."

It is important to emphasize that theories $\mathrm{A}, \mathrm{B}$ and $\mathrm{C}$ were not given to the learner as some sort of limited hypothesis space. Rather, the number of possible theories the learner could consider in each case is potentially infinite, but practically it settles on one of these three or their logical equivalents. Many other theories besides A, B and C were considered by the learner, but they do not figure significantly into the trajectory of learning. These theories are much less good (i.e., unnecessarily complex or poorly fitting) relative to neighboring knowledge states, so they tend to be proposed and accepted only in the early, more random stages of learning, and are quickly discarded. We could not find a way to group these other theories into cohesive or sensibly interpreted classes, and since they are only transient states of the learner, we removed them for purposes of analyzing learning curves and studied only the remaining proportions, renormalized.

In order to see how the dynamics of learning depend on data, consider specifically cases in which there are few magnetic objects that are not magnets, perhaps 1 or 2 (as in cases 1 and 2). In this case a partial theory such as theory A might suffice. According to this theory there is only one type of interacting object, and one law. If there are two magnetic non-magnets in the domain, the partial theory will classify them as 'interacting' objects based on their behavior with the magnets, conflating them with the magnets. However, it will incorrectly predict the two magnetic non-magnets should interact with each other. Their failure to interact will be treated as an outlier by the learner who has theory $\mathrm{A}$. The full theory $\mathrm{C}$ can correctly predict this non-interaction, but it does so by positing more laws and types of objects, which has a lower prior probability. As the number of magnetic non-magnets increases, the number of 'outliers' in the data increase as well (see Figure $8 \mathrm{~b})$. Theory A now predicts more and more incorrect interactions, and in a Kuhnian fashion there is a point at which these failures can no longer be ignored, and a qualitative shift to a new theory is preferred. In a completely different scenario, such as the extreme case of only 1 magnet (case X), we 
might expect the learner to not come up with magnet interactions laws, and settle instead on theory B.

For each one of the outlined cases we ran 70 simulations for 1600 iterations. Figure 8c shows the effect of data and time on the learning process, by displaying the relative proportion of the outlined theories at the end of the iteration for all simulations. Note the transition from case 1 to case 5: With a small number of non-magnet magnetic objects, the most frequently represented theory is theory A, which puts all magnetic objects (magnet or not) into a single class and treats the lack of interactions between two magnetic non-magnets as essentially noise. As the number of magnetic non-magnets increases, the lack of interactions between the different non-magnets can no longer be ignored and the full theory becomes more represented. Case X presents a special scenario in which there is only 1 magnet, and as expected theory $\mathrm{B}$ is the most represented there. The source of the difference between the proportion of theories learned in these different cases is the data the learner was exposed to. Within each case, the learner undergoes a process of learning similar to that described in the case studies - adopting and discarding theories in a process or time-driven manner.

To summarize, theory acquisition can be both data-driven and processdriven. Our simulations suggest that, at least in this simplified domain, both sufficient data and sufficient time to think are required. Only when the observed data provide a strong enough signal - as measured here by potential outliers under a simpler theory - is there sufficient inductive pressure for a Bayesian learner guided by simplicity priors to posit a more complex theory. Yet even with all the data in the world, a practical learning algorithm still requires sufficient time to think, time to search through a challenging combinatorial space of candidate laws and novel concepts and construct a sequence of progressively higher scoring theories that will reliably converge on the highest scoring theory for the domain. ${ }^{5}$ The fact that both sufficient data and sufficient time are needed for proper theory learning fits with the potentially frustrating experience of many teachers and parents: having laid out for a child all the data, all the input, that they need to solve a problem, grasp some explanation, or make a discovery, the child still doesn't seem to get it, or takes surprisingly long to get it. Knowing that any realistic learner

\footnotetext{
${ }^{5}$ It should however be noted that in some cases, the time-component allows the learner to 'weed out' and abandon overly complex theories.
} 
needs both data enough, and time, may at least provide some relief from that frustration and the patience to watch and wait as learning does its work.

\section{Evidence from experiments with children}

While our work here has been primarily motivated by theoretical concerns, we also want to consider the empirical evidence that children's learning corresponds in some way to the computational picture we have developed. Our most basic result is that a simple, cognitively plausible, stochastic search algorithm, guided by an appropriate grammar and language for theories, is capable of solving the rather sophisticated joint inference problem of learning both the concepts and the laws of a new theory - what we referred to as the "hard problem" or the "chicken-and-egg" problem of theory learning. In the last few years, several lines of experimental work have shown that children and adults can indeed solve this joint inference problem in the course of acquiring new theories. Kemp et al. (2010) showed that adults were able to learn new causal relations, such as objects of type A light up type $B$, and to use these relations to categorize objects, for example object 3 is of type $A$. In (Lucas et al., 2010) adults performed a task asking about specific causal structures leading to evidence (which objects are 'blickets' that cause a 'blicket-meter' to activate), which required inferring the abstract functional form of the causal relations (do blickets activate the meter via a noisy-OR function, a deterministic disjunctive function or a conjunctive function). A similar experiment (Lucas \& Griffiths, 2010) demonstrated that children are also able to acquire such abstract knowledge about the functional causal form while considering the specific identity of objects.

While in these studies children were explicitly told that only one type of concept is involved, Schulz and colleagues (Schulz et al., 2008) showed that young children can solve an even more challenging task: Given sparse evidence in the form of different blocks touching and making different noises, the children correctly posited the existence of three different causal kinds underlying the observed relations. In this case the children had to both infer the abstract relations governing the behavior, and posit how many concepts underly these relations. These papers are qualitatively consistent with our approach's predictions. In (Bonawitz et al., in press) we showed a more quantitative correspondence between our model predictions and children's categorization judgements. In that study children were shown interactions in a domain of simplified magnetism, where several unlabeled blocks interacted 
a.

Theory A

Rule 1: $\quad$ interacts $(X, Y) \longleftarrow f(X) \wedge f(Y)$

Theory B

Rule 1: $\quad$ interacts $(X, Y) \longleftarrow f(X) \wedge g(Y)$

Rule 2: $\quad$ interacts $(X, Y) \longleftarrow$ interacts $(Y, X)$

\section{Theory C}

Rule 1: $\quad$ interacts $(X, Y) \longleftarrow f(X) \wedge f(Y)$

Rule 2: $\quad$ interacts $(X, Y) \longleftarrow f(X) \wedge g(Y)$

Rule 3: $\quad$ interacts $(X, Y) \longleftarrow \operatorname{interacts}(Y, X)$ b.
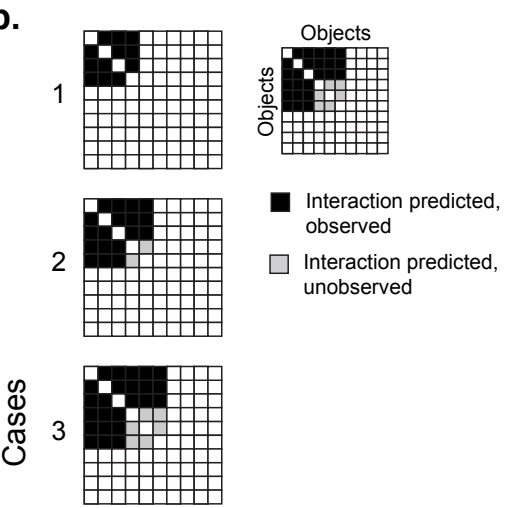

4
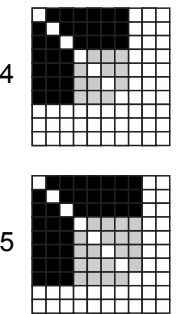

c.

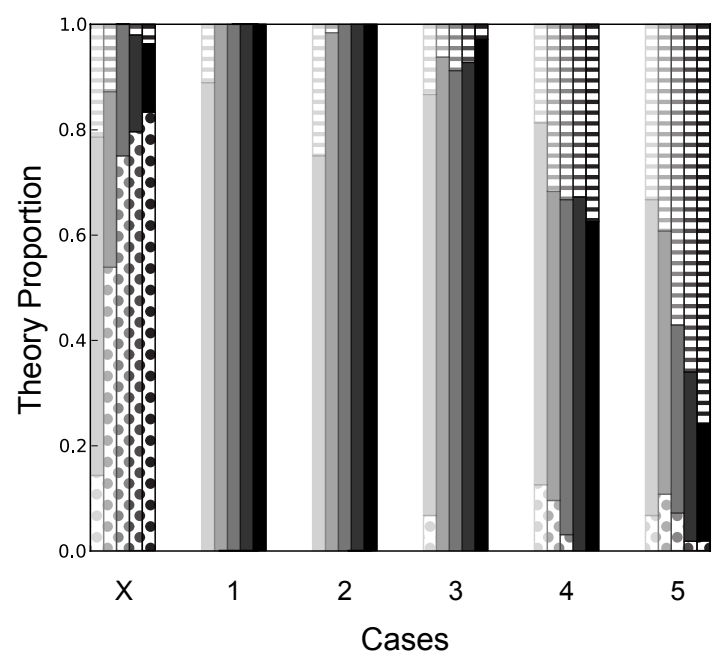

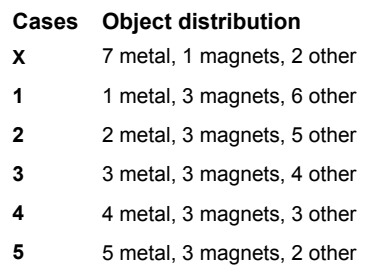

Theory A

Theory $B$

目 Theory C

Iterations

$\begin{array}{lllll}50 & 400 & 800 & 1200 & 1600\end{array}$

Figure 8: Learning dynamics resulting from two different sources: (a) A formal description of theories A, B and C (b) The predicted and observed interactions given theory A for the different cases, showing the growing number of outliers as the number of magnetic non-magnet objects grows (c) Proportion of theories accepted by the learner for different cases, during different points in the simulation runs. More opaque bars correspond to later iterations in the simulation. Different theories are acquired as a result of varying time and data. 
with blue and yellow blocks, either attracting or repelling from them. We also showed that the Monte Carlo search algorithm given here is capable of finding just the theories that children do, or theories that are behaviorally indistinguishable from them, and revising them appropriately.

Could models from an alternative paradigm such as connectionism also explain these results? Connectionist architectures could potentially solve aspects of the tasks described in (Lucas et al., 2010), for example. There are certainly networks capable of distinguishing between different functional forms like those in (Lucas et al., 2010), which may be seen as learning governing laws in a theory. Connectionist networks can also form new concepts - in the sense of clusters of data that behave similarly - via competitive learning. However, it has yet to be shown that a connectionist network can learn or represent the kinds of abstract knowledge that our approach does, and that children grasp in the other experiments cited above: solving the joint inference problem of discovering a system of new concepts and laws that together explain a set of previously unexpected interactions or relations. This problem poses an intriguing open challenge for connectionist modelers in cognitive development, one that could stimulate significant new research.

Going forward, we would like more fine-grained tests of whether and how the Monte Carlo search learning mechanism we have posited corresponds to the mechanisms by which children explore their space of theories. This will be challenging, as most of the steps of learning are not directly observable. We are currently working on studies together with Bonawitz and colleagues to test some general predictions of our model, such as the tradeoff between data and time described in the section above. In these experiments we recreate the domain of simplified magnetism described in the case studies section, with three types of objects that interact according to several laws. The children will be given different amounts of evidence, and crucially different segments of time, after which they will be asked to sort the objects they see into categories and describe why they do so. The children will not be told in advance how many object types exist, and we anticipate the number of types posited by the children will depend on their current domain theory. We anticipate the same amount of evidence but varying lengths of time will lead children to transition from one theory to the next, which will be evidenced in their sorting behavior. This behavior will be matched with running the stochastic search algorithm for varying amounts of time, as described in the previous section, though we recognize these are still only indirect tests of the model's predictions. 
More precision could come from microgenetic methods (Siegler \& Crowley, 1991), which study developmental change by giving children the same task several times and inspecting the strategies used to solve the task at many intervals. Microgenetic studies find that often, while the task itself remains constant, the strategies used to solve it undergo go. This data could be interpreted as a search process unfolding over time. A fundamental question for the microgenetic method remains why and how change occurs. Our algorithmic approach offers an explanation of how, and can potentially address the why. Together with Bonawitz and colleagues we are developing micro genetic methods to test whether children's learning can be explained in terms of Monte Carlo search.

One key challenge in designing a microgenetic study is defining an externally measurable sign of the internal cognitive mechanism of hypothesis testing and discovery. Similar to how microgenetic studies keep a task fixed, we intend to observe how children play and experiment with a given set of objects, without introduce new objects or any new data in the form of new interactions that haven't been observed before. As in classic microgenetic studies, we intend to ask the children questions and encourage them to talk out loud about their hypotheses in, order to probe the state of their search at more abstract levels of the theory. We can score the theories they uncover using computational tools, and observe whether the pattern of theories abandoned, adopted and uncovered fits with Monte Carlo search.

\section{Discussion and Conclusion}

We have presented an algorithmic model of theory learning in a hierarchical Bayesian framework and explored its dynamics in several case studies. We find encouraging the successful course of acquisition for several example theories, and the qualitative parallels with phenomena of human theory acquisition. These results suggest that previous "ideal learning" analyses of Bayesian theory acquisition can be approximately realized by a simple stochastic search algorithm and thus are likely well within the cognitive grasp

of child learners. It is also encouraging to think that state-of-the-art Monte Carlo methods used in Bayesian statistics and artificial intelligence to approximate ideal solutions to inductive inference problems might also illuminate the way that children learn. At this intersection point between the computational level and the algorithmic level of analysis, we showed that theory change is expected to be both data-driven and process-driven. This is an 
important theoretical distinction, but the psychological reality of these two sources of learning dynamics and their interaction needs to be further studied in experiments with children and adults.

While the main contributions of this paper are in addressing the algorithmics of theory acquisition, the 'how', the introduction of law templates provides some insight regarding 'what' the structure of children's knowledge might be, and the coupling between how we answer 'what?' and 'how?' questions of learning. On an algorithmic level, we found such templates to be crucial in allowing learning to converge on a reasonable timescale. On a computational level, these templates can be seen as generalizing useful abstract knowledge across domains, and providing high-level constraints that apply across all domain theories. The formal framework section did not directly treat where such templates come from, but it is possible to imagine that some of them are built in as overarching constraints on knowledge. More likely, though, they are themselves learned during the algorithmic acquisition process. An algorithmic grammar-based model can learn templates by abstracting successful rules from their particular domain instantiation. That is, if the model (or child) discovers a particularly useful rule involving a specific predicate such as "if $i s_{-} a(\mathrm{X}, \mathrm{Y})$ and $i s_{-} a(\mathrm{Y}, \mathrm{Z})$, then $i s_{-} a(\mathrm{X}, \mathrm{Z})$ ", then the specific predicate might be abstracted away to form the transitive template "if $F(X, Y)$ and $F(Y, Z)$, then $F(Y, Z)$ ". Learning this transitive template then allows its reuse in subsequent theory, and represents a highly abstract level of knowledge.

There are many ways in which our modeling work here can and should be extended in future studies. The algorithm we have explored is only one particular instance of a more general proposal for how stochastic search operating over a hierarchically structured hypothesis space can account for theory acquisition. The specific theories considered here were only highly simplified versions of the knowledge children have about real-world domains. Part of the reason that actual concepts and theories are richer and more complex is due to the fact that children have a much richer underlying language for representations. Horn clauses are expressive and suitable for capturing some knowledge structures, and in particular certain kinds of causal relations, but they are not enough. A potentially more suitable theory space would be built on a functional language, in which the laws are more similar to mathematical equations. Such a space would be harder to search through, but it would be much more expressive. A functional language of this sort would allow us to explore rich theories described in children, such as basic notions about 
objects and their interactions (Spelke, 1990), and the intuitive physics guide (Baillargeon, 1994) object behavior. Despite the need for a more expressive language, we expect the same basic phenomena found in the model domains considered here to be replicated in more complex models. Moving forward, a broader range of algorithmic approaches, stochastic as well as deterministic, need to be evaluated as both as behavioral models and as effective computational approximations to the theory search problem for larger domains.

Relative to previous Bayesian models of cognitive development that focused on only the computational level of analysis, this paper has emphasized algorithmic-level implementations of a hierarchical Bayesian computational theory, and the interplay between the computational and algorithmic levels. We have not discussed at all the level of neural implementation, but recent proposals by a number of authors argue that analogous stochastic-sampling ideas could plausibly be used to carry out Bayesian learning in the brain (Fiser et al., 2010). More generally, a "top-down" path to bridging levels of explanation in the study of mind and brain, starting with higher, more functional levels and moving down to lower, more mechanistic levels, appears most natural for Bayesian or other "reverse-engineering" approaches to cognitive modeling (Griffiths et al., 2010). Other paradigms for cognitive modeling adopt different ways to navigate the same hierarchy. Connectionist approaches, for instance, start from hypothesized constraints on neural representations (e.g., distributed codes) and learning mechanisms (e.g., errordriven learning) and move up from there, to see what higher-level phenomena emerge (McClelland et al., 2010). While we agree that actual biological mechanisms will ultimately be a central feature of any account of children's cognitive development, we are skeptical that this is the best place to start (Griffiths et al., 2010). The details of how the brain might represent or learn knowledge such as the abstract theories we consider here remain largely unknown, making a bottom-up emergent alternative to our approach hard to contemplate. In contrast, while our top-down approach has yet to make contact with neural phenomena, it has yielded real insights spanning levels. In moving from computational-level accounts to algorithms that explicitly (if approximately) implement the computational theory let us see plainly how the basic representations of children's theories could be acquired, and suggest explanations for otherwise puzzling features of the dynamics of learning in young children, as the consequences of efficient and effective algorithms for approximating the rational computational-level ideal of Bayesian learning. We hope that as neuroscience learns more about the neural substrates 
of symbolic representations and mechanisms of exploratory search, our topdown approach can be meaningfully extended from the algorithmic level to the level of implementation in the brain's hardware.

Going back to the puzzle of the "chicken and egg" problem posed at the beginning of the paper, what do the dynamics explored here tell us about the coupled challenges of learning the laws of a theory and the invention of truly novel concepts, and the opposing views represented by Fodor and Carey? There is a sense in which, at the computational level, the learner already must begin the learning process with all the laws and concepts needed to represent a theory already accessible. Otherwise the necessary hypothesis spaces and probability distributions for Bayesian learning could not be defined. In this sense, Fodor's skepticism on the prospects for learning or constructing truly novel concepts is justified. Learning cannot really involve the discovery of anything "new", but merely the changing of one's degree of belief in a theory, transporting probability mass from one part of the hypothesis space to another. However, on the algorithmic level explored in this paper, the level of active processing for any real-world learner, there is in fact genuine discovery of new concepts and laws. Our learning algorithm can begin with no explicitly represented knowledge in a given domain - no laws, no abstract concepts with any non-trivial extensions in the world - and acquire reasonable theories comprised of novel laws and concepts that are meaningfully grounded and predictively useful in that domain.

Our specific algorithm suggests the following account of how new concepts derive their meanings. Initially, the concepts themselves are only blank predicates. The theory prior induces a non-arbitrary structure on the space of possible laws relating these predicates, and in that sense can be said to contain a space of proto-meanings. The data are then fused with this structure in the prior to create a structured posterior: the concepts are naturally extended over the observed objects in those regions where the posterior has a high probability, and those are the areas in theory space that the learner will converge towards. This algorithmic process is, we suggest, an instance (albeit a very simple one) of Carey's "bootstrapping" account (Carey, 2004, 2009) of conceptual change, and a concrete computational implementation of concept learning under an inferential role semantics.

Under Carey's account of the origins of new concepts, children first use symbols as placeholders for new concepts and learn the relations between them that will support later inferential roles. Richer meaning is then filled in on top of these placeholders and relations, using a "modeling process" involv- 
ing a range of inductive inferences. The outer loop of our algorithm explains the first stage: why some symbolic structures are used rather than others and how their relations are created. The second stage of Carey's account parallels the inner loop of our algorithm, which attempts to find the likeliest and sparsest assignment of the core predicates, once their interactions have been fixed by the proposed theory. During our algorithmic learning process, new concepts may at times have only a vague meaning, especially when they are first proposed. Concepts that are fragmented can be unified, and concepts that are lumped together may be usefully dissociated, as learners move around theory space in ways similar to how new concepts are manipulated in both children's and scientists' theory change (Carey, 2009).

Returning to the overarching idea of the child as scientist, it is interesting to recall how from its inception, the study of the cognitive development of children was heavily influenced by the philosophy of science. Many researchers have found the metaphor of children as Lilliputian scientists useful and enlightening, seeing children as testing hypotheses and building structured causal models of the world, and this idea has found an exact formulation in an ideal Bayesian framework. However, neither children nor scientists are ideal, and discovering the practical learning algorithms of children may also lead us back to a better understanding of the process and dynamics of science itself as a search process.

Despite our optimism, it is important to end by stressing that our models at best only begin to capture some aspects of how children acquire their theories of the world. We agree very much with the view of Schulz (2012) that the hardest aspects of the problem are as yet unaddressed by any computational account, that there are key senses in which children's learning is a kind of exploration much more intelligent and sophisticated than even a smart randomized search such as our grammar-based MCMC. How could our learning algorithms account for children's sense of curiosity, knowing when and where to look for new evidence? How do children come up with the proper interventions to unconfound concepts or properties? How can a learning algorithm know when it is on the right track, so to speak, or distinguish good bad ideas from bad bad ideas, which children seem able to do? How do pedagogy and learning from others interact with interact with internal search dynamics are the ideas being taught simply accepted, or do they form the seed of a new search? How can algorithmic models go beyond the given evidence and actively explore, in the way children search for new data when appropriate? There is still much toil left - much rewarding toil, we hope - until we can say 
reasonably that we have found a model of children's learning, and believe it.

\section{Acknowledgments}

We wish to thank Laura Schulz, Liz Bonawitz, Alison Gopnik, Rebecca Saxe, Henry Wellman and Yarden Katz for helpful discussions. This work was funded by grants from the McDonnell Causal Learning Collaborative, ONR (N00014-09-0124), ARO (W911NF-08-1-0242) and an NSF Graduate Fellowship to the first author.

\section{Appendix A. Taxonomy data}

For the simulations described in Section 4.1 we used 7 objects (animal, bird, fish, canary, eagle, shark, salmon), and 7 properties (breathes, can_fly, can_swim, can_sing, has_claws, can_bite, is_pink). The core relations were set up as in (Katz et al., 2008). and used to generate the full set of true facts as the observable data:

$h a s_{-} a($ animal,breathes), has_a(bird,breathes), has_a(fish,breathes),

has_a(canary,breathes), has_a(eagle,breathes), has_a(shark,breathes),

$h a s_{-} a($ salmon,breathes), has_a(bird,can_fly), has_a(canary,can_fly),

$h a s_{-} a($ eagle,can_fly), has_a(fish,can_swim), has_a(shark,can_swim),

has_a(salmon,can_swim), has_a(canary,can_sing), has_a(eagle,has_claws),

has_a(shark,can_bite), has_a(salmon,is_pink),

$i s_{-} a($ animal,animal $), i s_{-} a($ bird,animal $), i s_{-} a($ fish,animal $)$,

$i s_{-} a($ bird,bird $), i s_{-} a($ fish,fish $), i s_{-} a($ canary,bird $)$,

$i s_{\_} a($ canary,animal $), i s_{-} a($ eagle,bird $), i s_{-} a($ eagle,animal $)$,

$i s_{-} a($ shark,fish $), i s_{-} a($ shark,animal $), i s_{-} a($ salmon,fish $)$,

$i s_{-} a($ salmon,animal $), i s_{-} a($ canary,canary $), i s_{-} a($ eagle,eagle $)$,

$i s \_a($ shark,shark $), i s \_a($ salmon,salmon $)$.

\section{Appendix B. Simplified magnetism data}

For the simulations described in Section 4.2 we used 10 objects: 3 magnets (objects 1-3), 5 magnetic objects (4-8) and two non-magnetic objects (9-10). The rules described in Section 4.2 were then used to generate an interaction matrix as the observable data:

Objects 1, 2, 3 each interact with objects $1,2,3,4,5,6,7,8$.

Objects 4, 5, 6, 7, 8 each interact with objects 1,2,3.

Objects 9, 10 do not interact with any other object. 


\section{References}

Baillargeon, R. (1994). How Do Infants Learn About the Physical World? Current Directions in Psychological Science, 3, 133-140.

Bishop, C. M. (2006). Pattern recognition and machine learning. (1st ed.). Springer.

Block, N. (1986). Advertisement for a Semantics for Psychology. Midwest Studies in Philosophy, 10, 615-678.

Bonawitz, E., Gopnik, A., Denison, S., \& Griffiths, T. (in press). Rational randomness: The role of sampling in an algorithmic account of preschooler's causal learning. In F. Xu, \& T. Kushnir (Eds.), Rational Constructivism. Elsevier.

Bradshaw, G., Langley, P., \& Simon, H. (1983). Studying scientific discovery by computer simulation. Science, 22, $971-975$.

Bruner, J. S., Goodnow, J. J., \& Austin, G. A. (1956). A study of thinking. New York: Wiley.

Carey, S. (1985). Conceptual change in childhood. Cambridge, MA: MIT Press/Bradford Books.

Carey, S. (2004). Bootstrapping and the origin of concepts. Daedalus, 133, $59-68$.

Carey, S. (2009). The Origin of Concepts. Oxford University Press.

Collins, A., \& Quillian, M. (1969). Retrieval time from semantic memory. Journal of Verbal Learning and Verbal Behavior, 8, 240-247.

Feldman, J. (2006). An algebra of human concept learning. Journal of Mathematical Psychology, 50, 339-368.

Feldman, J. A., Ps, J. G. I., Horning, J. J., Reder, S., Feldman, J. A., Gips, J., Horning, J. J., \& Reder, S. (1969). Grammatical complexity and inference. Technical Report Stanford University.

Field, H. (1977). Logic, Meaning, and Conceptual Role. Journal of Philosophy, 74, 379-409. 
Fiser, J., Berkes, P., Orbn, G., \& Lengye, M. (2010). Statistically optimal perception and learning: from behavior to neural representations. Trends in Cognitive Sciences, 14, 119 - 130.

Fodor, J., \& Lepore, E. (1991). Why meaning (probably) isn't conceptual role. Mind \& language, $6,328-343$.

Fodor, J. A. (1975). The language of thought. Harvard University Press: Cambridge, MA.

Fodor, J. A. (1980). On the impossibility of acquiring 'more powerful' structures. In Language and Learning: The Debate Between Jean Piaget and Noam Chomsky. Harvard University Press.

Fodor, J. A., \& Pylyshyn, Z. W. (1988). Connectionism and cognitive architecture: A critical analysis. Cognition, 28, 3-71.

Gershman, S., Vul, E., \& Tenenbaum, J. B. (2009). Perceptual multistability as Markov Chain Monte Carlo inference. Advances in Neural Information Processing Systems, 22, $611-619$.

Gilks, W., \& Spiegelhalter, D. (1996). Markov chain Monte Carlo in practice. Chapman \& Hall/CRC.

Goodman, N. D., Mansinghka, V. K., Roy, D. M., Bonawitz, K., \& Tenenbaum, J. B. (2008a). Church: a language for generative models. Uncertainty in Artificial Intelligence, .

Goodman, N. D., Tenenbaum, J. B., Feldman, J., \& Griffiths, T. L. (2008b). A rational analysis of rule-based concept learning. Cognitive Science, 32, $108-154$.

Goodman, N. D., Ullman, T. D., \& Tenenbaum, J. B. (2011). Learning a theory of causality. Psychological Review, 118, 110-119.

Gopnik, A., \& Meltzoff, A. N. (1997). Words, Thoughts, and Theories. Cambridge, MA: MIT Press.

Griffiths, T. L., Chater, N., Kemp, C., Perfors, A., \& Tenenbaum, J. B. (2010). Probabilistic models of cognition: exploring representations and inductive biases. Trends in Cognitive Sciences, 14, 357 - 364. 
Harman, G. (1975). Meaning and semantics. In M. Munitz, \& P. Unger (Eds.), Semantics and Philosophy. New York: New York University Press.

Harman, G. (1982). Conceptual role semantics. Notre Dame Journal of Formal Logic, 23, 242-257.

Katz, Y., Goodman, N. D., Kersting, K., Kemp, C., \& Tenenbaum, J. B. (2008). Modeling semantic cognition as logical dimensionality reduction. Proceedings of the Thirtieth Annual Conference of the Cognitive Science Society, .

Kemp, C., Goodman, N. D., \& Tenenbaum, J. B. (2007). Learning causal schemata. In Proceedings of the Twenty-ninth Annual Meeting of the Cognitive Science Society.

Kemp, C., Goodman, N. D., \& Tenenbaum, J. B. (2008a). Learning and using relational theories. Advances in Neural Information Processing Systems, 20,753-760.

Kemp, C., Goodman, N. D., \& Tenenbaum, J. B. (2008b). Theory acquisition and the language of thought. In Proceedings of Thirtieth Annual Meeting of the Cognitive Science Society.

Kemp, C., \& Tenenbaum, J. B. (2009). Structured statistical models of inductive reasoning. Psychological Review, 116, 20-58.

Kemp, C., Tenenbaum, J. B., Niyogi, S., \& Griffiths, T. L. (2010). A probabilistic model of theory formation. Cognition, 114, 165-196.

Kirkpatrick, S., Gelatt, C. D., \& Vecchi, M. P. (1983). Optimization by simulated annealing. Science, 220, 671-680.

Lucas, C. G., Gopnik, A., \& Griffiths, T. L. (2010). Learning the form of causal relationships using hierarchical bayesian models. In Proceedings of the 32nd Annual Conference of the Cognitive Science Society.

Lucas, C. G., \& Griffiths, T. L. (2010). Developmental differences in learning the forms of causal relationships. Cognitive Science, 34, 113-147.

Marr, D. (1982). Vision. Freeman Publishers. 
McClelland, J. L., Botvinick, M. M., Noelle, D. C., Plaut, D. C., Rogers, T. T., Seidenberg, M. S., \& Smith, L. B. (2010). Letting structure emerge: connectionist and dynamical systems approaches to cognition. Trends in Cognitive Sciences, 14, $348-356$.

Mcclelland, J. L., \& Rumelhart, D. E. (Eds.) (1986). Parallel Distributed Processing: Explorations in the Microstructure of Cognition, Volume 2: Psychological and Biological Models. Cambridge, MA: MIT Press.

Mitchell, T. (1982). Generalization as search. Artificial Intelligence, (pp. 203-226).

Moreno-Bote, R., Knill, D., \& Pouget, A. (2011). Bayesian sampling in visual perception. Proceedings of the National Academy of Sciences, 108, $12491-12496$.

Murphy, G. L., \& Medin, D. L. (1985). The role of theories in conceptual coherence. Psychol Rev, 92, 289-316.

Newell, A., \& Simon, H. (1976). Computer science as empirical inquiry: Symbols and search. Communications of the ACM, 19, 113-126.

Pearl, J. (2000). Causality: models, reasoning, and inference. Cambridge University Press.

Rogers, T. T., \& McClelland, J. L. (2004). Semantic cognition: A parallel distributed processing approach. Cambridge, MA: MIT Press.

Russell, S., \& Norvig, P. (2009). Artificial Intelligence: a modern approach. (3rd ed.). Prentice Hall.

Schulz, L. E. (2012). Finding new facts; thinking new thoughts. In Rational Constructivism, Advances in Child Development and Behavior. Elsevier volume 42.

Schulz, L. E. (In press). The origins of inquiry: Inductive inference and exploration in early childhood. Trends in Cognitive Science, .

Schulz, L. E., Goodman, N. D., Tenenbaum, J. B., \& Jenkins, A. C. (2008). Going beyond the evidence: abstract laws and preschoolers' responses to anomalous data. Cognition, 109, 211-223. 
Shultz, T. R. (2003). Cognitive Developmental Psychology. Cambridge, MA, USA: MIT Press.

Siegler, R., \& Crowley, K. (1991). The micro genetic method. American Psychologist, 46, 606-620.

Siegler, R. S., \& Chen, Z. (1998). Developmental differences in rule learning: A microgenetic analysis. Cognitive Psychology, 36, 273-310.

Smith, E., \& Medin, D. (1981). Categories and Concepts. Cambridge, MA: Harvard University Press.

Spall, J. C. (2003). Introduction to stochastic search and optimization: Estimation, simulation, and control. John Wiley and Sons.

Spelke, E. (1990). Principles of object perception. Cognitive Science, 14, $29-56$.

Spirtes, P., Glymour, C., \& Scheines, R. (2001). Causation, Prediction, and Search. (2nd ed.). Cambridge, MA, USA: MIT Press.

Sundareswara, R., \& Schrater, P. R. (2008). Perceptual multistability predicted by search model for bayesian decisions. Journal of Vision, 8 .

Tenenbaum, J. B., \& Griffiths, T. L. (2001). The rational basis of representativeness. In Proceedings of the 23rd Annual Conference of the Cognitive Science Society (pp. 1036-1041).

Tenenbaum, J. B., Griffiths, T. L., \& Kemp, C. (2006). Theory-based bayesian models of inductive learning and reasoning. Trends in Cognitive Sciences, 10, 309-318.

Tenenbaum, J. B., Griffiths, T. L., \& Niyogi, S. (2007). Intuitive theories as grammars for causal inference. In A. Gopnik, \& L. Schulz (Eds.), Causal learning: Psychology, philosophy, and computation. Oxford: Oxford University Press.

Tenenbaum, J. B., Kemp, C., Griffiths, T. L., \& Goodman, N. D. (2011). How to grow a mind: Statistics, structure, and abstraction. Science, 331, 1279-1285. 
Wellman, H. M., Fang, F., \& Peterson, C. C. (2011). Sequential Progressions in a Theory-of-Mind Scale: Longitudinal Perspectives. Child Development, 82, 780-792.

Wellman, H. M., \& Gelman, S. A. (1992). Cognitive development: Foundational theories of core domains. Annual Review of Psychology, 43, 337-375.

Wellman, H. M., \& Woolley, J. D. (1990). From simple desires to ordinary beliefs: The early development of everyday psychology. Cognition, 35, 245-275. 